\title{
A combined Fourier-Bessel transformation method to derive accurate rotational velocities
}

\author{
A.J.M. Piters ${ }^{1}$, P.J. Groot ${ }^{1}$ and J. van Paradijs ${ }^{1,2}$ \\ 1 Astronomical Institute 'Anton Pannekoek' / CHEAF, Kruislaan 403, NL-1098 SJ Amsterdam, The Netherlands \\ 2 Physics Department UAH, Huntsville, AL 35899, U.S.A.
}

Received November 22, 1995; accepted January 23, 1996

\begin{abstract}
We describe in some detail the characteristics of a combined Fourier-Bessel transformation technique to derive projected equatorial rotational velocities from spectral line profiles. This technique shares with the Fourier-transformation method, developed by Gray, that it distinguishes rotational broadening of a spectral line from broadening by other mechanisms. The range of rotational velocity values that can be derived with this method is limited mainly by the spectral resolution (low velocities) and by line blending and the signal-to-noise ratio (high velocities). We discuss the uncertainty on the outcoming rotational velocity as a result of various effects, such as limbdarkening, spectral resolution, noise, data-preparation, and intrinsic broadening. We conclude that the Fourier-Bessel transformation method can provide rotational velocities, with a typical uncertainty down to a few percent. It does not include any modelling of individual stars with effects as anisotropic macroturbulence included and therefore is less suited for a detailed analysis of individual stars. It is suited for statistical investigation of a large sample of stars.
\end{abstract}

Key words: methods: data analysis — methods: numerical

\section{Introduction}

In this paper we discuss the characteristics of a method to derive projected equatorial rotational velocities from spectral absorption lines. This method was introduced by Deeming (1977), who showed that a combined FourierBessel transform of a line profile shows a peak, the location of which is determined by the rotational velocity. The aim of this paper is to investigate the working range of this method and its limiting conditions. In Sect. 2.1 we present the theoretical basis of this method; the effects of other (not rotational) broadening mechanisms are investigated in Sect. 2.2, the effect of the Fourier-frequency range that is chosen for the Bessel transform in Sect. 2.3, the effects of data sampling in Sect. 2.4. In Sect. 3 we present some practical considerations in the application of this method on real spectral lines. We discuss and summarize our results in Sect. 4.

\section{The method}

\subsection{Rotational broadening}

The method of Fourier-Bessel transformation of spectral lines, as introduced by Deeming (1977), is based on the philosophy that an integral transform is optimal when

Send offprint requests to: A.J.M. Piters the transformed signal is close to a delta function. The Fourier-Bessel transform of a purely rotationally broadened line profile is such a delta function.

A spectral line emitted from a uniformly emitting spherical star without limb darkening, and which undergoes no other broadening mechanisms, has an ellipsoidal profile $I_{\text {rot }}(\Delta \lambda)$ :

$$
I_{\text {rot }}(\Delta \lambda)= \begin{cases}\frac{2}{\pi b} \sqrt{1-\left(\frac{\Delta \lambda}{b}\right)^{2}}, & ,|\Delta \lambda| \leq b \\ 0 & ,|\Delta \lambda|>b,\end{cases}
$$

(see, e.g., Shajn \& Struve 1920; Carrol 1933). Here $\Delta \mathrm{t}$ is the wavelength difference $\lambda-\lambda_{0}, \lambda_{0}$ is the central wavelength of the line profile and the parameter $b$ is proportional to the projected rotational velocity $v \sin i$ :

$$
b=\lambda_{0} \frac{v \sin i}{c} .
$$

Note that throughout this paper the capital $I$ is used to indicate a line profile, and not a specific intensity.

The Fourier transform $F_{\text {rot }}(u)$, with $u$ in cycles per wavelength unit $\left(\AA^{-1}\right)$, of the profile given by Eq. (1), is proportional to a first order Bessel function, scaled with the Fourier-frequency $u$

$$
F_{\text {rot }}(u) \equiv \int_{-\infty}^{\infty} I_{\text {rot }}(\Delta \lambda) \mathrm{e}^{2 \pi i u \Delta \lambda} \mathrm{d} \Delta \lambda=\frac{J_{1}(2 \pi u b)}{\pi u b} .
$$


The Bessel transform $B_{\text {rot }}(s)$, with $s$ in $\AA$, of the Fourier transform $F_{\text {rot }}(u)$ is the Fourier-Bessel transform of the line profile

$$
B_{\mathrm{rot}}(s) \equiv \int_{0}^{\infty} 2 \pi u^{2} F_{\mathrm{rot}}(u) J_{1}(2 \pi u s) \mathrm{d} u=\frac{2}{b^{2}} \delta(b-s) .
$$

Hence, the Fourier-Bessel transformation is a delta function with a peak at $s=b$. So for a line with the "ideal" rotational profile (Eq. 1) the rotational velocity is, with Eq. (2), directly obtained from the position of the maximum of the Fourier-Bessel transform (Eq. 4). Note that, in principle, Eq. (4) has the disadvantage that noise at high frequencies in $\mathrm{F}$ is weighted relatively strong by the $u^{2}$ factor. Of course, whether or not, in pratice, this is a problem depends on the frequency to which the integration extends (see Sect. 2.3) and on the $S / N$-ratio of the spectral data used. In our application of this method to a sample of $\sim 200 \mathrm{~F}$ dwarfs (see Groot et al. 1996, hereafter Paper II), we found that this was not a problem. The extra factor $u^{2}$ however is important and necessary to make an orthogonal set of functions.

\subsection{Other broadening mechanisms}

In more realistic spectral lines other mechanisms will also play a role in the broadening of the line profile. This can influence the shape of the Fourier-Bessel transform of the spectral line and, more importantly, the position of its maximum. In this subsection we investigate the effects of broadening mechanisms that result in a Voigt profile. A Voigt profile is a convolution of a Gauss profile $I_{\mathrm{G}}(\Delta \mathrm{\lambda})$ (e.g., Doppler broadening) and a Lorentz profile $I_{\mathrm{L}}(\Delta \mathrm{\lambda})$ (e.g., damping). We assume that the Gauss and Lorentz broadening profiles are isotropic.

The line profile $I(\Delta \lambda)$ is then given by:

$$
\begin{aligned}
I(\Delta \lambda) & =\left(I_{\mathrm{G}} * I_{\mathrm{L}} * I_{\text {rot }}\right)(\Delta \lambda) . \\
\text { Here } & \\
I_{\mathrm{G}}(\Delta \lambda) & =\frac{1}{\sqrt{\pi} \Delta \lambda_{\mathrm{D}}} \mathrm{e}^{-\left(\Delta \mathrm{\lambda} / \Delta \mathrm{\lambda}_{\mathrm{D}}\right)^{2}}, \\
I_{\mathrm{L}}(\Delta \lambda) & =\frac{\Delta \lambda_{\mathrm{H}}}{\pi}\left(\Delta \lambda^{2}+\frac{1}{4} \Delta \lambda_{\mathrm{H}}^{2}\right)^{-1},
\end{aligned}
$$

and, $\Delta \lambda_{\mathrm{H}}$ is the full width at half maximum of the Lorentz profile and $\Delta \lambda_{\mathrm{D}}$ is the Gaussian line broadening parameter equivalent to the Doppler width. Also, we have assumed the line to be not too strong, such that saturation effects are absent. The effect of saturation was already investigated by Deeming (1977).

The Fourier transform $F(u)$ of this rotationally broadened Voigt profile is the product of the Fourier transforms of the contributing profiles $F_{\mathrm{G}}(u), F_{\mathrm{L}}(u)$ and $F_{\text {rot }}(u)$ :

$$
F(u)=\int_{-\infty}^{\infty} I(\Delta \lambda) \mathrm{e}^{2 \pi i u \Delta \lambda} \mathrm{d} \Delta \lambda
$$

$$
\begin{aligned}
& =F_{\mathrm{G}}(u) \cdot F_{\mathrm{L}}(u) \cdot F_{\mathrm{rot}}(u) \\
& =2 \mathrm{e}^{-\left(\pi u \Delta \lambda_{\mathrm{D}}\right)^{2}} \mathrm{e}^{-\pi u \Delta \lambda_{\mathrm{H}}} \frac{J_{1}(2 \pi u b)}{\pi u b} .
\end{aligned}
$$

The Bessel transform $B(s)$ of the Fourier transform is then given by:

$$
B(s)=\int_{0}^{\infty} \frac{4 u}{b} J_{1}(2 \pi u s) J_{1}(2 \pi u b) \mathrm{e}^{-\left(\pi u \Delta \lambda_{\mathrm{D}}\right)^{2}} \mathrm{e}^{-\pi u \Delta \lambda_{\mathrm{H}}} \mathrm{d} u .
$$

Because the integral simplifies considerably if we let either $\Delta \lambda_{\mathrm{H}}=0$ or $\Delta \mathrm{\lambda}_{\mathrm{D}}=0$, the influence of the Gaussian and exponential factors to the Bessel transform is investigated separately.

\subsubsection{Gaussian profile}

First, we consider the case without damping, $\Delta \lambda_{\mathrm{H}}=0$. The integral (Eq. 8) then reduces to

$$
B(s)=\frac{2}{b\left(\pi \Delta \lambda_{\mathrm{D}}\right)^{2}} \exp \left(-\frac{b^{2}+s^{2}}{\Delta \lambda_{\mathrm{D}}^{2}}\right) I_{1}\left(\frac{2 b s}{\Delta \lambda_{\mathrm{D}}^{2}}\right),
$$

where $I_{n}$ is the modified Bessel function defined by $I_{n}(x)=(-i)^{n} J_{n}(i x)$. The Bessel transform $B(s)$ is shown in Fig. 1, for Doppler widths ranging between $\Delta \mathrm{M}_{\mathrm{D}}=0.1 b$ and $0.8 b$. The maximum of $B(s)$ can be found by calculating the roots of its derivative. We find that the derivative $\mathrm{d} B(s) / \mathrm{d} s$ is zero if

$$
I_{0}(x)-\left(\frac{1}{x}+a x\right) I_{1}(x)=0,
$$

where $x=2 b s / \Delta \lambda_{\mathrm{D}}^{2}$ and $a=\Delta \lambda_{\mathrm{D}}^{2} / 2 b^{2}$, so that $a x=s / b$. Here, we have used the general property of Bessel functions that $\mathrm{d} I_{\nu}(z) / \mathrm{d} z=I_{\nu-1}(z)-\nu I_{\nu}(z) / z$.

So for a particular value of the parameter $a$, which is directly related to the line broadening parameter $\Delta \lambda_{D}$, Eq. (10) defines the value $s_{\max }$ of $s=a x \cdot b$ for which the Bessel transform $B(s)$ reaches its maximum. Figure 2 shows the value of $s_{\max } / b$ as a function of $\Delta \lambda_{\mathrm{D}} / b$.

If there is no broadening by other mechanisms than rotation $\left(\Delta \lambda_{\mathrm{D}}=0\right)$, the Bessel transform will reach its maximum at $s_{\max }=b$, which can be seen from Eq. (10) by calculating the limit for very large values of $x$ (i.e. very small values of $\left.\Delta \lambda_{\mathrm{D}}\right)$, so that $I_{0}(x) \approx I_{1}(x)$ :

$$
\lim _{\Delta \Lambda_{\mathrm{D}} \rightarrow 0} \frac{s_{\max }}{b}=\lim _{x \rightarrow \infty} a x=\lim _{x \rightarrow \infty} \frac{I_{0}(x)}{I_{1}(x)}-\frac{1}{x}=1 .
$$

For very large values of $\Delta \lambda_{\mathrm{D}}, s$ approaches $\frac{1}{2} \sqrt{2} \Delta \lambda_{\mathrm{D}}$ (dashed line), as follows from:

$$
\lim _{\Delta \lambda_{\mathrm{D}} \rightarrow \infty} \frac{2 s_{\max }^{2}}{\Delta \lambda_{\mathrm{D}}^{2}}=\lim _{x \rightarrow 0} a x^{2}=\lim _{x \rightarrow 0} \frac{I_{0}(x)}{I_{1}(x)} x-1=1 .
$$

The dip in the function $s_{\max }\left(\Delta \lambda_{\mathrm{D}}\right)$ around $\Delta \lambda_{\mathrm{D}}=0.77 b$ (Fig. 2) can be understood from the relation for the derivative of this function:

$$
\frac{\mathrm{d} s_{\max }}{\mathrm{d} \Delta \lambda_{\mathrm{D}}}=\sqrt{2 a} x \frac{a^{2} x^{4}+(a-1) x^{2}-1}{a^{2} x^{4}+(2 a-1) x^{2}-1} .
$$




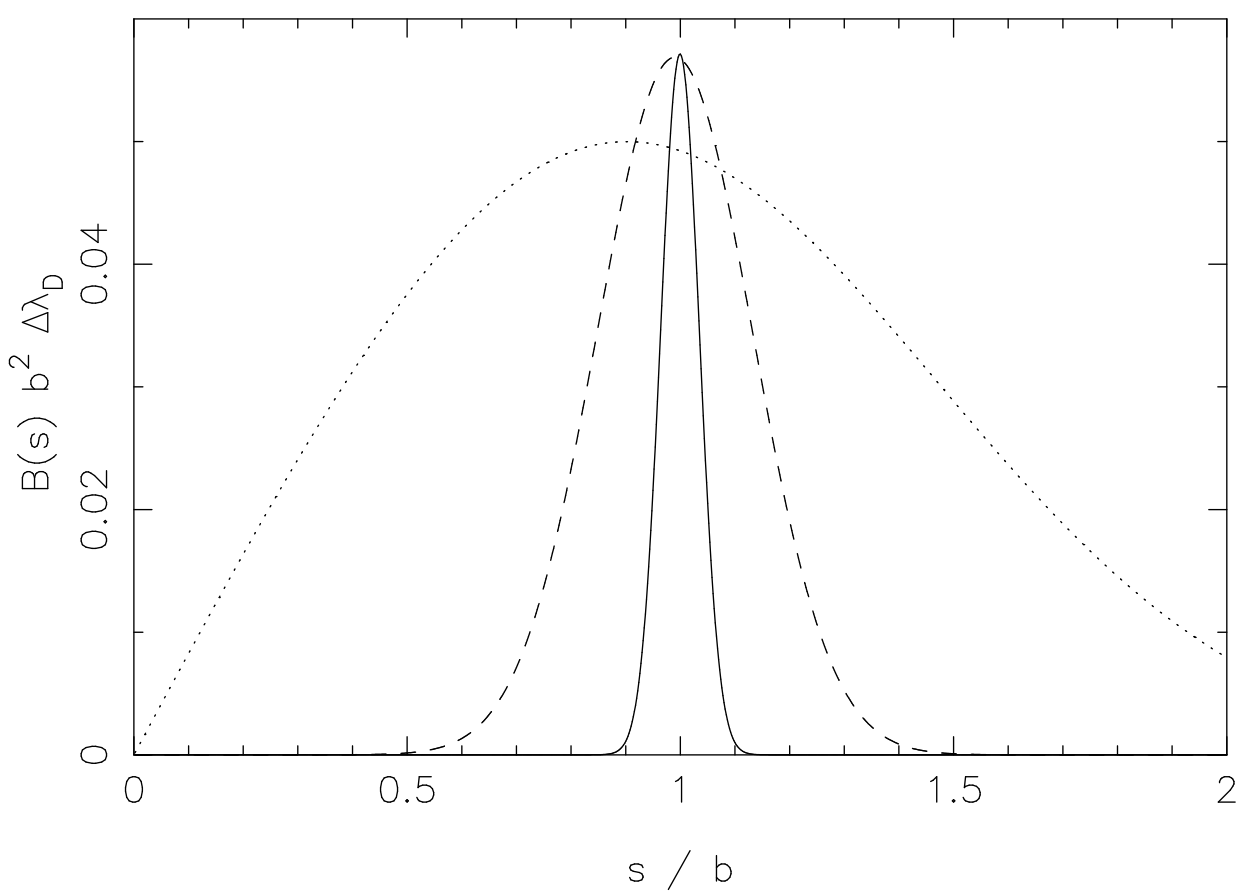

Fig. 1. The Fourier-Bessel transform $B(s)$ in units of $b^{-2} \Delta \lambda_{\mathrm{D}}^{-1}$, as a function of the parameter $s$ in units of $b$, for different values of the Doppler width $\Delta \lambda_{\mathrm{D}}$. The solid line is for $\Delta \lambda_{\mathrm{D}}=0.1 b$, the dashed line for $\Delta \lambda_{\mathrm{D}}=0.2 b$, and the dotted line for $\Delta \lambda_{\mathrm{D}}=0.8 b$

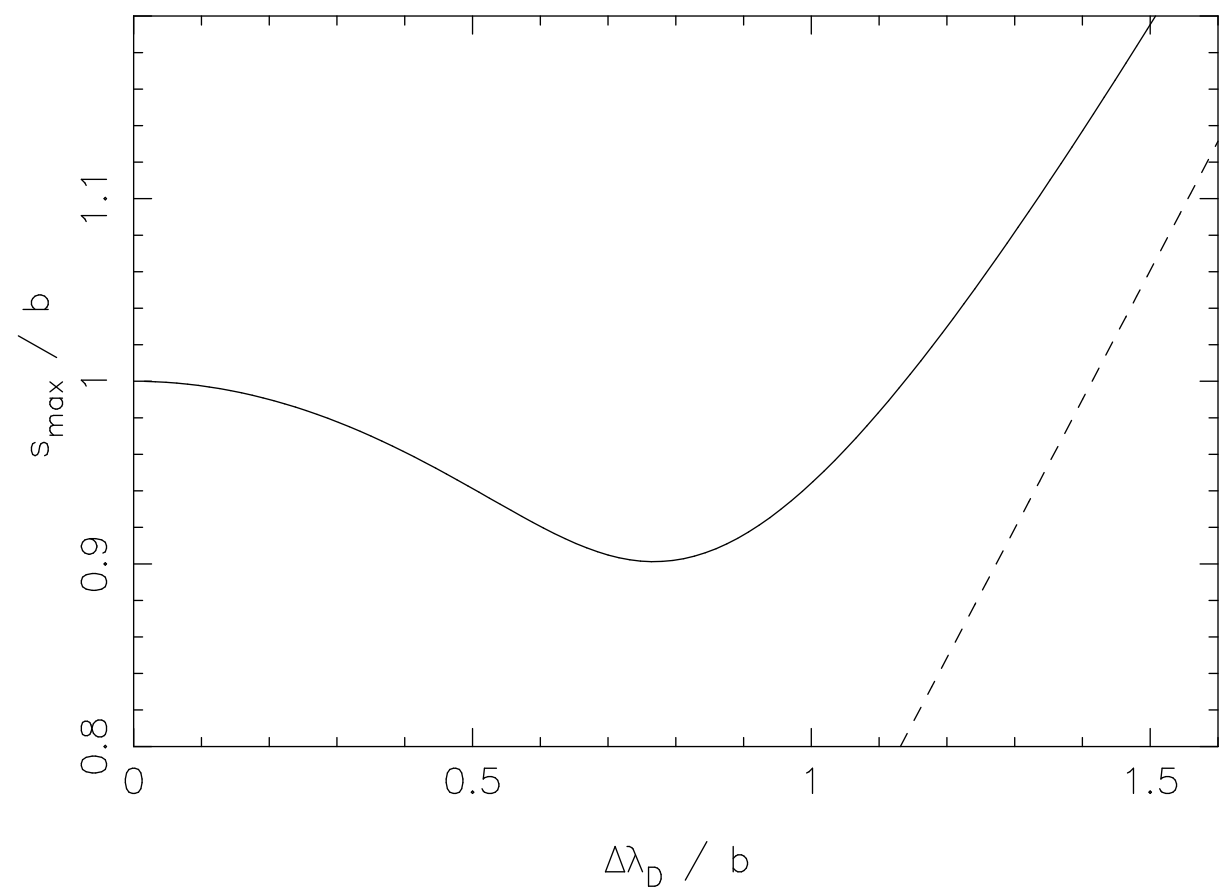

Fig. 2. The location of the maximum of the Fourier-Bessel transform $s_{\max }$, in units of the rotational velocity parameter $b$, as a function of the width of the Gaussian broadening $\Delta \lambda_{\mathrm{D}}$, also in units of $b . s_{\max }$ differs from $b$ by less than $1 \%$ if $\Delta \lambda_{\mathrm{D}}<0.2 b$ and less than $10 \%$ if $\Delta \lambda_{\mathrm{D}}<1.3 b$. The dashed line gives the limit where $s$ approaches $\frac{1}{2} \sqrt{2} \Delta \lambda_{\mathrm{D}}$

This derivative is zero for $x \rightarrow \infty$, (and, with Eq. (11), With Eq. (10) this criterion becomes: $a \rightarrow 0, a x=1$ and $\left.\Delta \lambda_{\mathrm{D}}=0\right)$. The derivative is also zero if the pair $(a, x)$ follow the criterion $a^{2} x^{4}+(a-1) x^{2}-1=0$.

$$
\left(\left(\frac{I_{0}(x)}{I_{1}(x)}\right)^{2}-1\right) x^{2}-\frac{I_{0}(x)}{I_{1}(x)} x-1=0 .
$$


This equation has exactly one solution, which is around $x \approx 3.1$, hence $\Delta \mathrm{U}_{\mathrm{D}} \approx 0.77 b$, explaining the minimum in Fig. 2 at this value.

Figure 2 shows that the maximum of the Besseltransform differs from $b=\lambda_{0} v \sin i / c$ by less than $1 \%$ as long as $\Delta \lambda_{\mathrm{D}}<0.2 b$ and by less than $10 \%$ as long as $\Delta \Lambda_{\mathrm{D}}<1.3 b$. This means that for e.g. a $v_{\text {turb }} \simeq 10 \mathrm{~km} / \mathrm{s}$ (average speed of sound in $\mathrm{F}$ dwarfs), the error is less than $1 \%$ for $v_{\text {rot }} \sin i \geq 50 \mathrm{~km} / \mathrm{s}$ and less than $10 \%$ for $v_{\text {rot }} \sin i \geq$ $8 \mathrm{~km} / \mathrm{s}$.

\subsubsection{Lorentz profile}

Second, we study the effect of broadening mechanisms which result in a Lorentz profile, e.g., damping. For this purpose we substitute $\Delta \lambda_{D}=0$ in Eq. (8), which then reduces to:

$$
B(s)=\frac{3}{16 \sqrt{2} \pi^{2}} \frac{\Delta \lambda_{\mathrm{H}}}{(b s)^{3 / 2}} \frac{1}{z^{5 / 2}} F\left(\frac{7}{4}, \frac{5}{4} ; 2 ; \frac{1}{z^{2}}\right),
$$

where $F(a, b ; c ; z)$ is a hypergeometric function (Oberhettinger 1970), and $z=\left(\frac{1}{4} \Delta \Lambda_{\mathrm{H}}^{2}+b^{2}+s^{2}\right) / 2 b s$.

The maximum $s_{\max }$ of $B(s)$ is reached when $s$ follows the relation:

$$
\frac{\left(s^{\prime}-z\right)}{\left(5 s^{\prime}-2 z\right)}=-\frac{8}{35} z^{2} \frac{F\left(\frac{7}{4}, \frac{5}{4} ; 2 ; \frac{1}{z^{2}}\right)}{F\left(\frac{11}{4}, \frac{9}{4} ; 3 ; \frac{1}{z^{2}}\right)},
$$

where $s^{\prime}=s / b$.

Figure 3 shows the value of $s_{\max } / b$, for which $B(s)$ reaches its maximum, as a function of $\Delta \lambda_{H} / b$. For very large values of $\Delta \lambda_{\mathrm{H}}$, the hypergeometric functions approach unity, hence the value for $s_{\max } / b$ approaches the dashed line, which is defined by the relation:

$$
\frac{16}{35} z^{3}-\frac{8}{7} s^{\prime} z^{2}+z-s^{\prime}=0 .
$$

The fraction $F\left(\frac{7}{4}, \frac{5}{4} ; 2 ; \frac{1}{z^{2}}\right) / F\left(\frac{11}{4}, \frac{9}{4} ; 3 ; \frac{1}{z^{2}}\right)$ in Eq. (16) approaches zero if $z$ approaches 1 , which can only occur for $s=b$ and $\Delta \lambda_{\mathrm{H}}=0$. So $s_{\max }=b, \Delta \lambda_{\mathrm{H}}=0$ is a solution of Eq. (16). The minimum in the function $s_{\max }\left(\Delta \lambda_{\mathrm{H}}\right)$ is reached at $\Delta \lambda_{\mathrm{H}} \approx 1.5 b$, where the value of $s_{\max }$ is $5 \%$ less than for $\Delta \lambda_{\mathrm{H}}=0$.

The maximum of the Bessel-transform differs from $b=$ $\lambda_{0} v \sin i / c$ by less than $1 \%$ as long as $\Delta \lambda_{\mathrm{H}}<0.4 b$ and less than $10 \%$ as long as $\Delta \lambda_{\mathrm{H}}<3 b$. Note that in general $\Delta \mathrm{\lambda}_{\mathrm{H}} \simeq 0.1 \Delta \mathrm{\lambda}_{\mathrm{D}}$.

\subsection{Cut-off frequency}

In evaluating the Bessel-transform we need to choose a cut-off frequency in the Fourier-domain (Deeming 1977). We find that the choice of this frequency is very important in the exact location of the maximum, in contrast with the findings of Deeming. Let us for now assume that $\Delta \lambda_{D}=0$ and $\Delta \lambda_{\mathrm{H}}=0$, so that the line profile is only broadened by rotation. Equation (8) then becomes:

$$
B_{u_{\mathrm{c}}}(s)=\int_{0}^{u_{\mathrm{c}}} \frac{2 u}{b} J_{1}(2 \pi u b) J_{1}(2 \pi u s) \mathrm{d} u,
$$

where $u_{\mathrm{c}}$ is the cut-off frequency. For $u_{\mathrm{c}} \rightarrow \infty$, the Besseltransform is a delta function: $B_{\infty}(s)=2 \delta(s-b) / b^{2}$.

Figure 4 shows the value of $s$ where the Besseltransform reaches its maximum, $s_{\max }$, as a function of the cut-off frequency (solid line). The vertical dashed lines give the positions of the zero-points of the Bessel-function $J_{1}\left(2 \pi u_{\mathrm{c}} b\right)$. These lines intersect the curve $s_{\max }\left(u_{\mathrm{c}}\right)$ at local minima in this curve, which can be seen by studying the relation for the derivative of $s_{\max }\left(u_{\mathrm{c}}\right)$ with respect to $u_{\mathrm{c}}$ :

$$
\begin{aligned}
\frac{\mathrm{d} s_{\max }}{\mathrm{d} u_{\mathrm{c}}} & =-\frac{\partial^{2} B(s)}{\partial u_{\mathrm{c}} \partial s} / \frac{\partial^{2} B(s)}{\partial s^{2}} \\
& =-\frac{u_{\mathrm{c}} J_{1}\left(2 \pi u_{\mathrm{c}} b\right) \frac{\partial J_{1}\left(2 \pi u_{\mathrm{c}} s\right)}{\partial s}}{\int_{0}^{u_{\mathrm{c}}}\left(\frac{u}{s^{2}}-4 \pi^{2} u^{3}\right) J_{1}(2 \pi u b) J_{1}(2 \pi u s) \mathrm{d} u}
\end{aligned}
$$

The derivative of $s_{\max }\left(u_{\mathrm{c}}\right)$ is zero if $J_{1}\left(2 \pi u_{\mathrm{c}} b\right)=0$, which is at the locations of the vertical dashed lines. The derivative is also zero if $\partial J_{1}\left(2 \pi u_{\mathrm{c}} s\right) / \partial s=0$ (the dot-dashed lines). The values of $s_{\max }$ in these local maxima are only slightly larger than $b$, with a maximum difference of $0.4 \%$ in the first maximum around $u_{\mathrm{c}} b=0.85$. Therefore these maxima can be used to estimate the rotational velocity.

The cut-off frequencies where $s_{\max }=b$ can be found by evaluating the derivative of the Bessel-transform at $s=b$, and requiring that this derivative be zero:

$$
\lim _{s \rightarrow b} \frac{\partial B_{u_{\mathrm{c}}}(s)}{\partial s}=\frac{u_{\mathrm{c}}^{2}}{2 b} J_{0}\left(2 \pi u_{\mathrm{c}} b\right) J_{2}\left(2 \pi u_{\mathrm{c}} b\right)=0,
$$

where we used that if $\lim _{x \rightarrow x_{0}} f(x)=0$ and $\lim _{x \rightarrow x_{0}} g(x)=0$, and $h(x)=f(x) / g(x)$, then:

$$
\lim _{x \rightarrow x_{0}} \frac{\mathrm{d} h(x)}{\mathrm{d} x}=\left.\frac{f^{\prime \prime}(x) g^{\prime}(x)-f^{\prime}(x) g^{\prime \prime}(x)}{2\left(g^{\prime}(x)\right)^{2}}\right|_{x=x_{0}} .
$$

Equation (20) is fulfilled where $J_{0}\left(2 \pi u_{\mathrm{c}} b\right)=0$ or $J_{2}\left(2 \pi u_{\mathrm{c}} b\right)=0$. Figure 4 shows the lines $J_{0}\left(2 \pi u_{\mathrm{c}} s\right)=0$ as the dash-dotted lines to the right of the local maxima and the lines $J_{2}\left(2 \pi u_{\mathrm{c}} s\right)=0$ as dotted lines to the left of the local maxima. For large values of the cutoff frequency these lines approach each other because $\lim _{x \rightarrow \infty} J_{2}(x)+J_{0}(x)=0$. The dot-dashed lines that intersect the local maxima of the curve $s_{\max }\left(u_{\mathrm{c}}\right)$ are given by the relation $\partial J_{1}\left(2 \pi u_{\mathrm{c}} s\right) / \partial s=0$, which is equivalent to the relation $J_{0}\left(2 \pi u_{\mathrm{c}} s\right)=J_{2}\left(2 \pi u_{\mathrm{c}} s\right)$, so that the local maxima of the curve $s_{\max }\left(u_{\mathrm{c}}\right)$ always lie between the intersections with the lines $J_{0}\left(2 \pi u_{\mathrm{c}} s\right)=0$ and $J_{2}\left(2 \pi u_{\mathrm{c}} s\right)=0$, and hence the values of the maxima approach $b$ if $u_{\mathrm{c}} \rightarrow \infty$.

The values of the local maxima for small cut-off frequencies can be found by substituting $\partial J_{1}\left(2 \pi u_{\mathrm{c}} s\right) / \partial s=0$ 


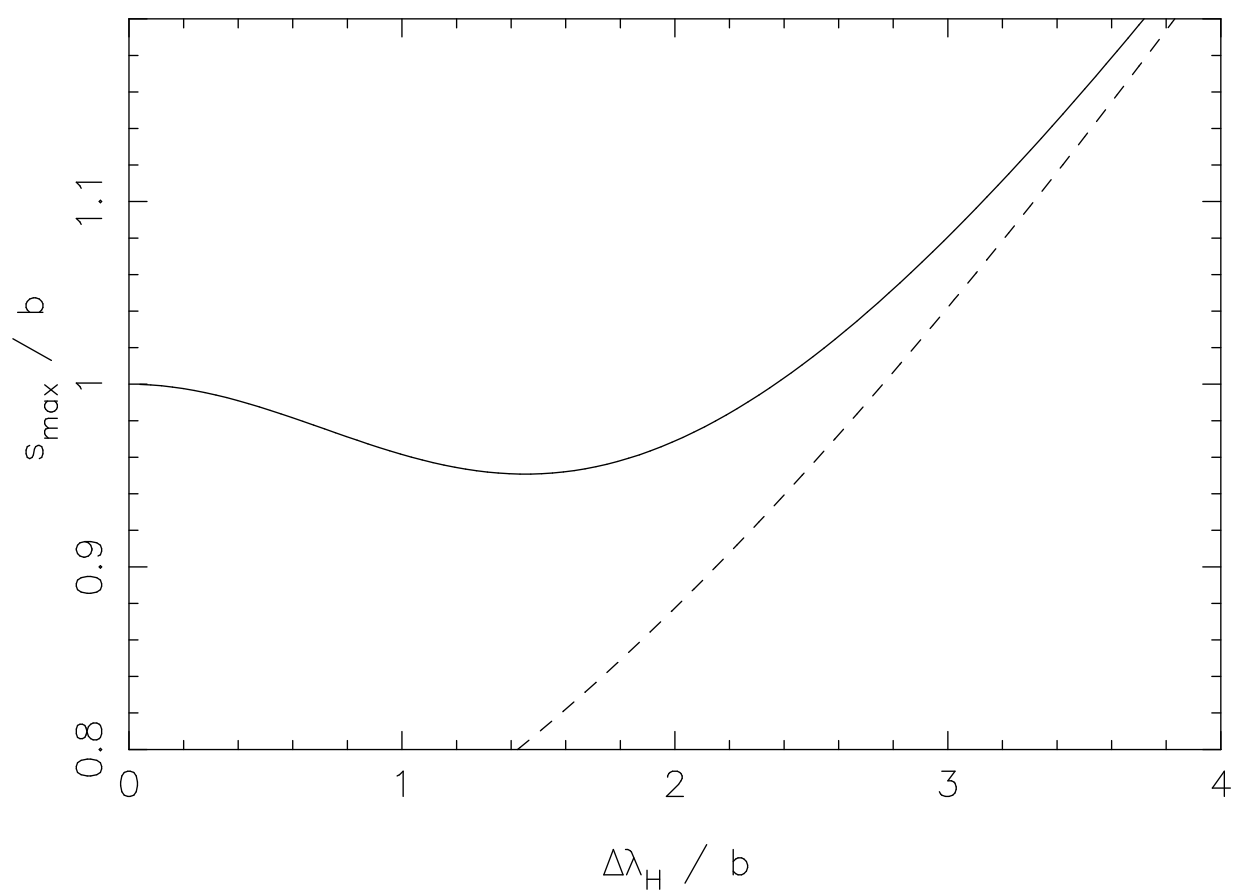

Fig. 3. The location of the maximum of the Fourier-Bessel transform $s_{\max }$, in units of the rotational velocity parameter $b$, as a function of the width of the Lorentz broadening $\Delta \lambda_{\mathrm{H}}$, also in units of $b . s_{\max }$ differs from $b$ by less than $1 \%$ if $\Delta \lambda_{\mathrm{H}}<0.4 b$ and less than $10 \%$ if $\Delta \lambda_{\mathrm{H}}<3 b$

in the derivative of the Bessel-transform, and requiring that this derivative be zero, which is equivalent to:

$$
\left(c^{4}-c^{2} x^{2}+x^{2}+c^{2}\right) J_{1}(x)=2 x c^{2} J_{0}(x),
$$

where $x=2 \pi u_{\mathrm{c}} b$ and $c$ is given by $J_{0}(c)=J_{2}(c)$. The values of the maxima are slightly larger than $b$ :

$$
\frac{s_{\max }-b}{b} \approx 410^{-3}, 610^{-4}, 1.610^{-4}, 610^{-5}, \ldots
$$

for increasing values of $u_{\mathrm{c}}\left(u_{\mathrm{c}} b=0.8485,1.3586,1.8631\right.$, $2.3656, \ldots)$. We may conclude therefore that in this ideal case the effect of a finite cut-off frequency is negligible $(<0.5 \%)$ compared with the errors introduced by other effects, such as noise, limb darkening etc. It is therefore possible to determine the projected rotational velocity of star by taking the height of the first local maximum in a figure like Fig. 4. In Paper II, we show an application of the FBT method in which the projected rotational velocities of $\sim 200 \mathrm{~F}$ dwarfs are determined in this way. Note that it is not necessary to choose a cut-off frequency in the Bessel transform in this way.

If other broadening mechanisms are included, the positions of the local maxima in the curve $s_{\max }\left(u_{\mathrm{c}}\right)$ are also determined by the relation $J_{2}\left(2 \pi u_{\mathrm{c}} s\right)=J_{0}\left(2 \pi u_{\mathrm{c}} s\right)$. This means that the local maxima of the $s_{\max }\left(u_{\mathrm{c}}\right)$ curve lie on the dot-dashed lines in Fig. 4, so the locations of these maxima depend slightly on their heights. The heights of the first 7 local maxima are shown in Fig. 5a for
Gaussian broadening and in Fig. 5b for Lorentz broadening, as a function of $\Delta \lambda_{\mathrm{D}} / b$ and $\Delta \lambda_{\mathrm{H}} / b$ respectively. This Figure shows that for cut-off frequencies that reach only the first local maximum (which is used in pratice, see Paper II) the deviation with respect to the results for an infinite cut-off frequency, is less than $1 \%$ and as the cut-off frequency increases, the maxima approach the asymptotic values from Figs. 2 and 3.

\subsection{Limb darkening}

The effect of limb darkening is a deformation of the elliptical rotation profile. For a linear limb-darkening law, $I(\theta) / I(0)=$ constant $\cdot(1+\beta \cos \theta)$, the Fourier transform of the rotation profile is (Böhm 1952):

$$
\begin{aligned}
F_{\text {rot }, \beta}(u) & =\frac{1}{\frac{1}{2}+\frac{\beta}{3}}\left\{\frac{J_{1}(2 \pi u b)}{2 \pi u b}-\right. \\
& \left.-\beta\left(\frac{\cos (2 \pi u b)}{(2 \pi u b)^{2}}-\frac{\sin (2 \pi u b)}{(2 \pi u b)^{3}}\right)\right\},
\end{aligned}
$$

and the Bessel transform of this is

$$
B_{\operatorname{rot}, \beta}(s)=\left\{\begin{array}{rr}
\frac{\beta}{\left(\frac{1}{2}+\frac{\beta}{3}\right)} \frac{s}{(2 \pi b)^{2}}\left\{\frac{1}{b \sqrt{b^{2}-s^{2}}+b^{2}-s^{2}}+\right. \\
\left.+\frac{1}{b \sqrt{b^{2}-s^{2}}+b^{2}}\right\}, \quad s<b \\
0 \quad, s>b
\end{array}\right.
$$




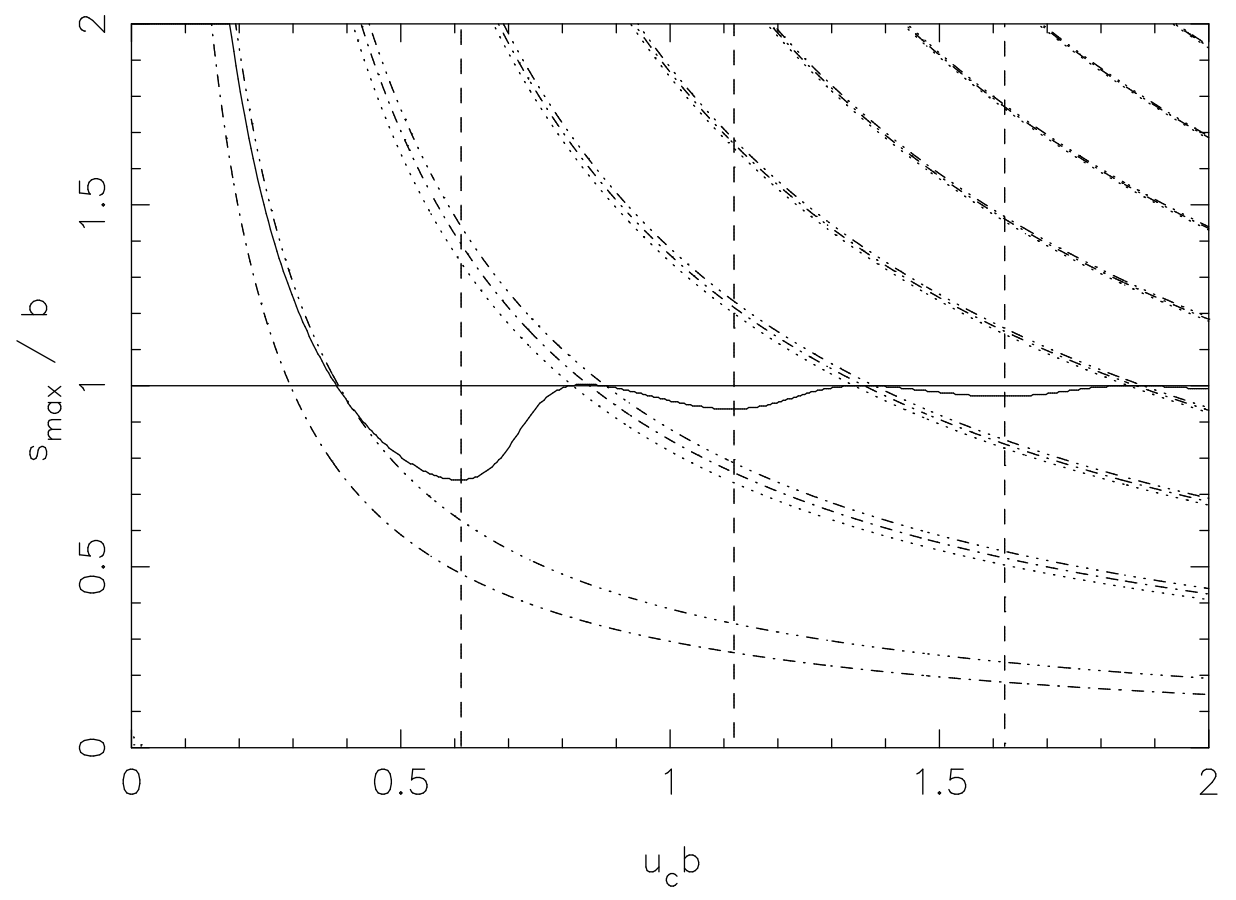

Fig. 4. The location of the maximum of the Fourier-Bessel transform $s_{\max }$, in units of the rotational velocity parameter $b$, as a function of the cut-off frequency $u_{\mathrm{c}}$, in units of $b^{-1}$ (solid line). Vertical dashed lines are defined by $J_{1}\left(2 \pi u_{\mathrm{c}} b\right)=0$, and intersect the local minima of the $s_{\max }\left(u_{\mathrm{c}}\right)$ curve. The series of three curves that go through or close through the local maxima of the $s_{\max }\left(u_{\mathrm{c}}\right)$ curve are defined by $J_{2}\left(2 \pi u_{\mathrm{c}} s\right)=0$ (left; dotted lines), $J_{0}\left(2 \pi u_{\mathrm{c}} s\right)=0$ (right; dash-dotted lines), and $\partial J_{1}\left(2 \pi u_{\mathrm{c}} s\right) / \partial s=0$ (middle; dot-dashed lines). The middle curve of these three defines the local maximum of the $s_{\max }\left(u_{\mathrm{c}}\right)$ curve, while the left and right curve intersect the $s_{\max }\left(u_{\mathrm{c}}\right)$ curve where the maximum of the Fourier-Bessel transform $s_{\max }$ is equal to the rotational velocity parameter $b$. For large values of the cut-off frequency $u_{\mathrm{c}}$, these three lines coincide, i.e., the local maxima of the $s_{\mathrm{max}}\left(u_{\mathrm{c}}\right)$ curve approach the rotational velocity parameter $b$

This transform is shown in Fig. 6, for different limbdarkening coefficients $\beta$. We see that the effect of limb darkening is a broadening of the delta function towards lower values of $s / b$. For $s$ larger than the rotational velocity parameter $b$, there is no contribution to the Besseltransform, while for $s$ less than $b$, it goes to infinity when $s$ approaches the parameter $b$. In the case of a purely rotationally broadened profile, deformed by a constant limb-darkening coefficient $\beta$, the maximum of the Besseltransform still occurs at $s=b$. But for more realistic lines, with intrinsic broadening, the effect of limb-darkening will be a shift of the maximum of the Bessel transform towards lower rotational velocities as can be expected, since the equatorial edges of the star, which have the largest radial velocities contribute relatively less when there is limb darkening. The magnitude of this effect is investigated in Sect. 3.2.

\subsection{Sampling}

The preceding sections dealt with Fourier-Bessel transforms of continuous functions. For observed spectral lines we know only the function value in discrete wavelength bins on a finite wavelength range. In this subsection we investigate the effect of sampling on the Fourier-Bessel transform. The flux is binned into $N$ wavelength intervals with width $\delta \lambda$, which gives a line profile $\tilde{I}_{\text {rot }}(\Delta \lambda)$ :

$$
\tilde{I}(\Delta \lambda)=\left\{\begin{array}{lc}
\frac{1}{\delta \lambda} \int_{\Delta \mathrm{\lambda}_{j}-\frac{1}{2} \delta \lambda}^{\Delta \mathrm{\lambda}_{j}+\frac{1}{2} \delta \lambda} I\left(\Delta \lambda^{\prime}\right) \mathrm{d} \Delta \lambda^{\prime} \\
0 & , \Delta \lambda=\Delta \lambda_{j}, j=1, \ldots, N \\
0 & , \text { otherwise }
\end{array}\right.
$$

where $\Delta \lambda_{j}$ is the central wavelength of bin $j$.

In the case of a purely rotationally broadened profile, the Fourier transform becomes:

$$
\tilde{F}_{\text {rot }}(u)=\frac{1}{\pi \delta \lambda} \sum_{j=1}^{N} \cos \left(2 \pi u \Delta \mathrm{\lambda}_{j}\right)\left[\theta-\frac{1}{2} \sin 2 \theta\right]_{\theta=\theta_{1, j}}^{\theta=\theta_{2, j}}
$$

where the integration boundaries $\theta_{1, j}$ and $\theta_{2, j}$ are given by:

$$
\begin{aligned}
\theta_{1, j} & =\arccos \frac{\Delta \lambda_{j}+\frac{1}{2} \delta \lambda}{b} \\
\theta_{2, j} & =\arccos \frac{\Delta \lambda_{j}-\frac{1}{2} \delta \lambda}{b}
\end{aligned}
$$



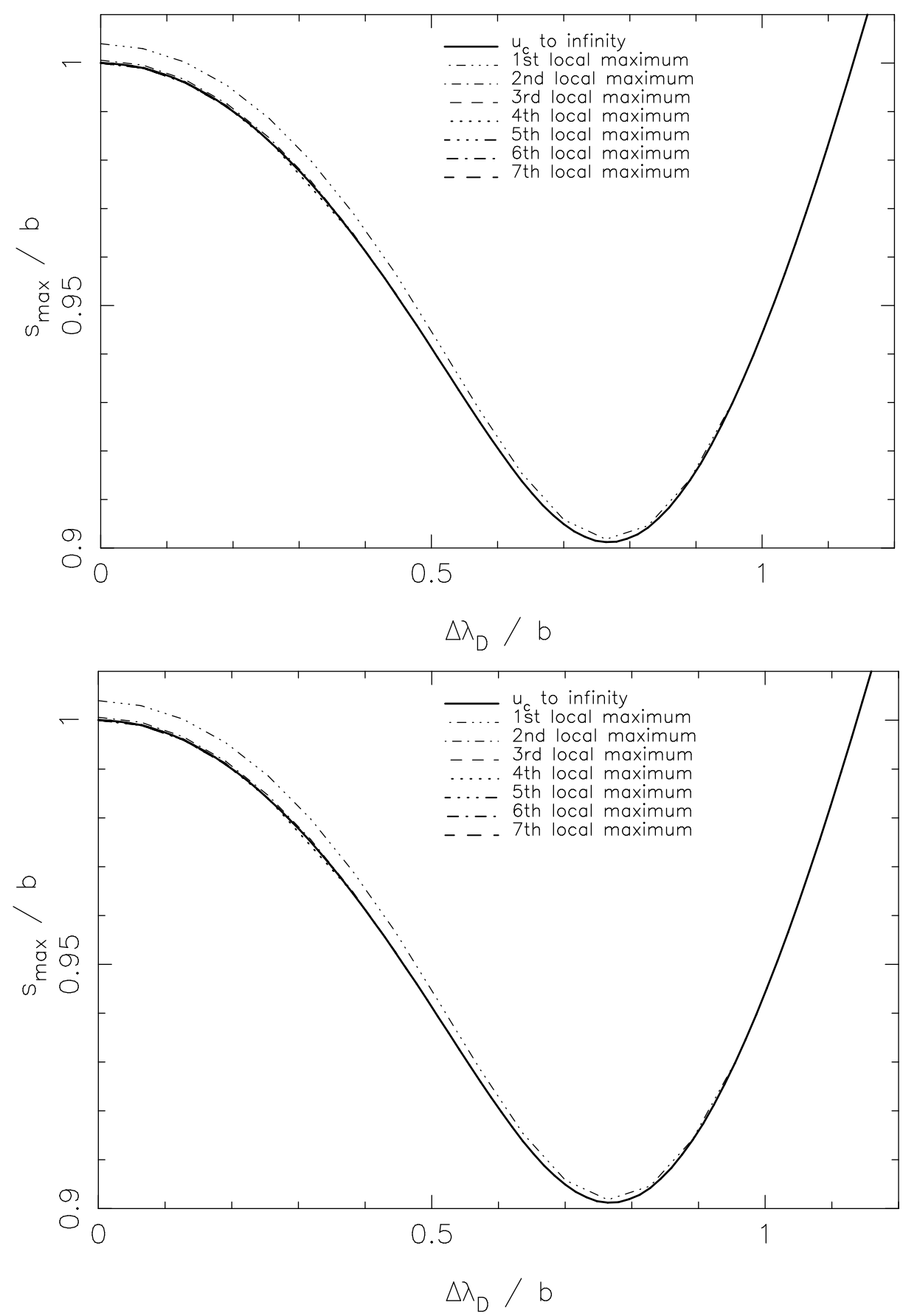

Fig. 5. The location of the maximum of the Fourier-Bessel transform $s_{\max }$, in units of the rotational velocity parameter $b$, for different values of the cut-off frequency $u_{\mathrm{c}}$ and as a function of the width of the Lorentz broadening $\left.\Delta \lambda_{\mathrm{D}} \mathbf{a}\right)$ and $\Delta \lambda_{\mathrm{H}} \mathbf{b}$ ), in units of $b$. Solid lines are for $u_{\mathrm{c}}=\infty$ and are the same as in Figs. 2 and 3 respectively. The other lines are for $u_{\mathrm{c}}$ at a local maximum of the $s_{\max }\left(u_{\mathrm{c}}\right)$ curve from Fig. 4 , as indicated in the figure

The range of frequencies over which the Bessel transform is performed is necessarily limited by the Nyquist frequency $u_{\mathrm{Nyq}}=1 /(2 \delta \lambda)$, because the sampling introduces a duplication of the Fourier transform at frequencies larger than $u_{\mathrm{Nyq}}$ :

$$
\begin{aligned}
\tilde{B}_{\text {rot }}(s)= & \frac{2}{\delta \lambda} \sum_{j=1}^{N}\left[\theta-\frac{1}{2} \sin 2 \theta\right]_{\theta=\theta_{1, j}}^{\theta=\theta_{2, j}} \\
& \cdot \int_{0}^{u_{\mathrm{Nyq}}} u^{2} J_{1}(2 \pi u s) \cos \left(2 \pi u \Delta \mathrm{U}_{j}\right) \mathrm{d} u
\end{aligned}
$$




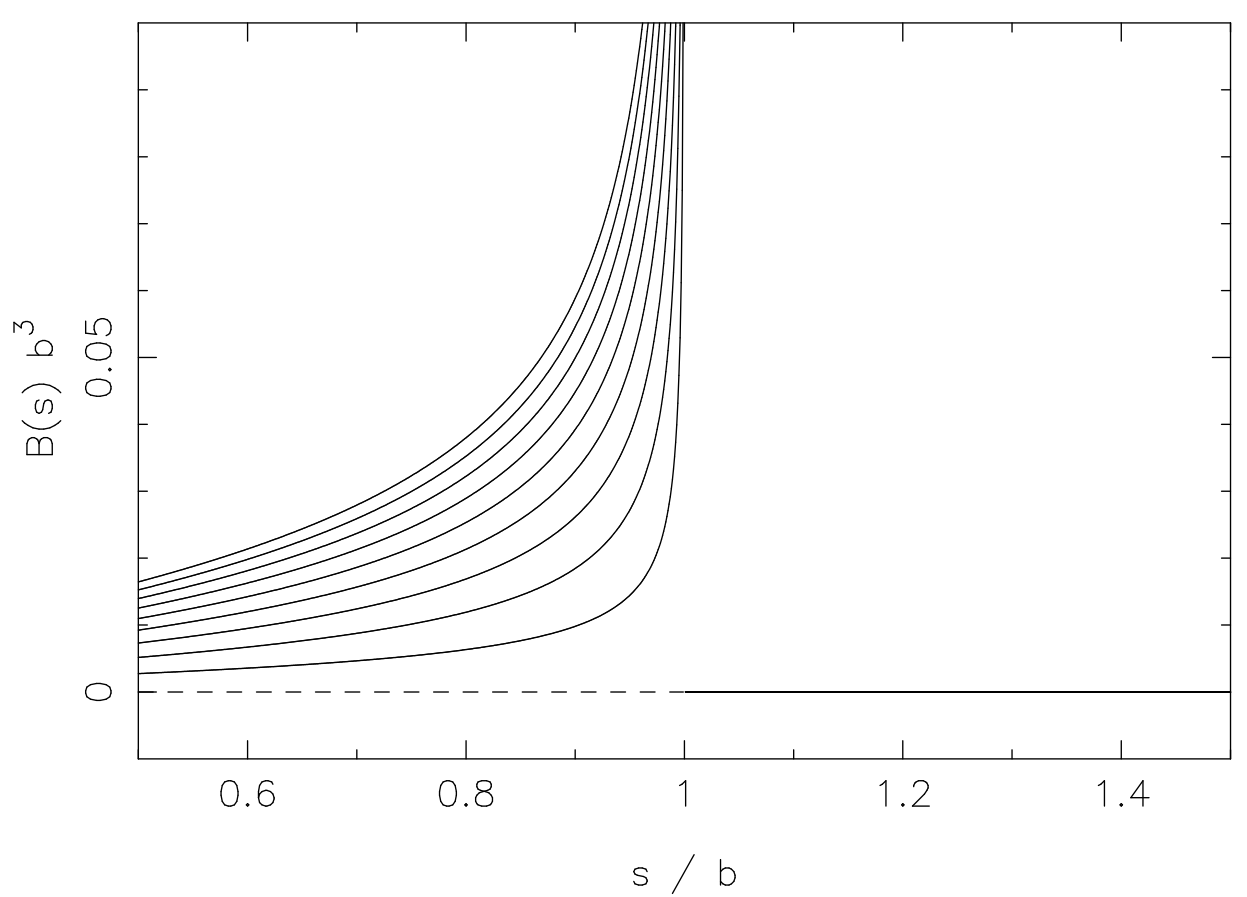

Fig. 6. The Fourier-Bessel transform $B(s)$, in units of $b^{-3}$, of a rotation profile, deformed by limb-darkening, for different values of the limb-darkening coefficient $\beta$. The value in $s=b$ is not defined. For $s>b$ the Fourier-Bessel transform is equal to zero, for $s<b$ the Fourier-Bessel transform is not zero. The dashed line denotes the Fourier-Bessel transform if there is no limb-darkening (its value is everywhere zero, except in $s=b$ ). The solid lines are, from bottom to top, for $\beta=0.1,0.2, \ldots, 0.9$ respectively

Figure 7 shows where the Bessel transform reaches its maximum as a function of the bin width $\delta \lambda$, for different values of the cut-off frequency, chosen to lie at local maxima of the $s_{\max }\left(u_{\mathrm{c}}\right)$ curve. From this Figure we see that the uncertainty in the rotational velocity increases for increasing wavelength bin width and for increasing cut-off frequency. The uncertainty is less than $1 \%$ if the bin width $\delta \lambda$ is less than $0.25 \mathrm{~b}$ (using a cut-off frequency of $1.36 / b$ ), i.e., if the rotation profile contains more than 8 wavelength bins. The uncertainty is less than $10 \%$, as long as the cut-off frequency does not exceed the Nyquist-frequency $1 /(2 \delta \lambda)$.

Windowing of the input profile needs not to be considered separately because the rotation profile, Eq. (1), is intrinsically confined, so that the Fourier transform over the wavelength range $[-\infty, \infty]$ is equivalent to the Fourier transform over the range $[-b, b]$.

\section{Practical considerations}

In the previous section we presented some analytical properties of the Fourier-Bessel transformation method, by isolating mechanisms that influence the position of the maximum of the transform. In practical applications all of the considered mechanisms can occur at the same time.

\subsection{Observational constraints}

The influence of observational uncertainties on the position of the maximum of the Fourier-Bessel transform of a spectral line can be minimized by obtaining observations with a high spectral resolution, as was shown in Sect. 2.5, and with a high signal-to-noise ratio, as we will show hereafter. The spectral lines need to be selected with great care: they should be clean lines (no blends), neither too weak, nor too strong (saturation; Deeming 1977), and preferably more than one spectral line should be used to determine the rotational velocity.

The position of the maximum in the Bessel transform is slightly influenced by noise. The noise variance on a general transform is proportional to the noise variance on the original data, in the common case of white noise on the input data. However, the noise on the Fourier-Bessel transform may be correlated (Deeming 1977). Figure 8 shows the average offset (dashed line) and the root mean square value (dot-dashed line) of the noise on the Bessel transform, assuming a purely rotationally broadened line profile with Poisson noise. The average and spread are calculated using a Monte Carlo simulation, i.e., we adopted the profile as given by Eq. (1) and chose an average number of counts per wavelength bin, $N=2500$ in the continuum, a bin width $(\delta \lambda=0.02 b)$, a relative central line intensity $r_{\mathrm{c}}=0.8$ (i.e., a relative line depth of 0.2 ), a wavelength 


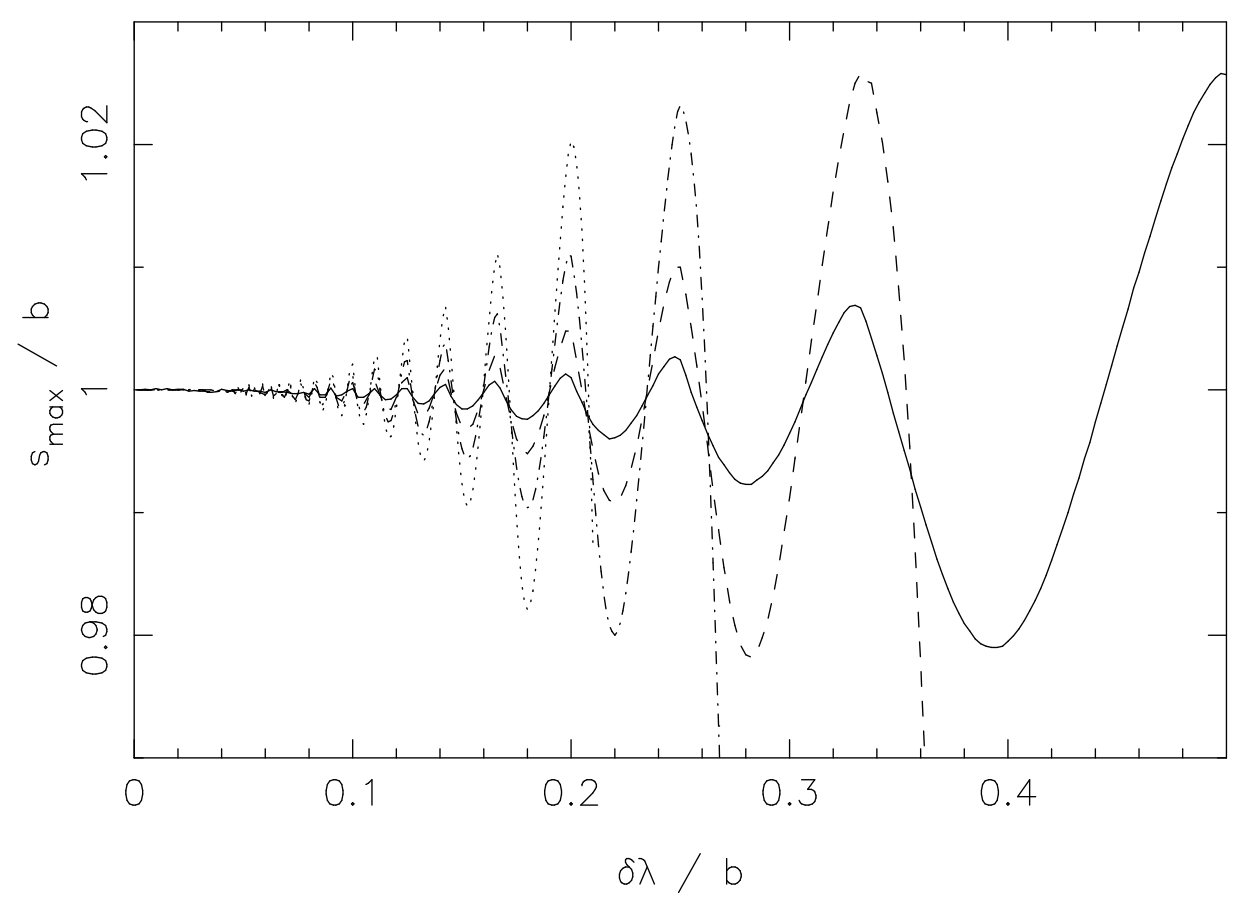

Fig. 7. The location of the maximum of the Fourier-Bessel transform $s_{\max }$, in units of the rotational velocity parameter $b$, as a function of the wavelength bin width $\delta \lambda$, also in units of $b$. Different lines denote different cut-off frequencies: the solid line is for a cut-off frequency $u_{\mathrm{c}}=0.85 / \mathrm{b}$, the dashed line for $u_{\mathrm{c}}=1.36 / \mathrm{b}$, the dot-dashed line for $u_{\mathrm{c}}=1.86 / \mathrm{b}$, and the dotted line for $u_{\mathrm{c}}=2.37 / b$. Lines are drawn until the point where the Nyquist frequency equals the cut-off frequency, i.e. where $\delta \lambda / b=1 /\left(2 u_{\mathrm{c}} b\right)$

range $W=\left[\lambda_{0}-b, \lambda_{0}+b\right]$ (representative of the data used in Paper II), and a cut-off frequency of $u_{\mathrm{c}} b=3.37$ (the 6th local maximum of the $s_{\max }\left(u_{\mathrm{c}}\right)$ curve in Fig. 4), and then selected the noise randomly according to a Poisson distribution. We repeated this noise selection 1000 times and calculated for every noisy profile the Fourier-Bessel transform. From the resulting 1000 profiles the average offset and the root mean square value are derived.

It follows from Fig. 8 that the average offset due to noise (dashed line) in the Fourier-Bessel transform is approximately zero, and that the root mean square of the noise (dot-dashed line) decreases as the value of $s$ increases.

We tested the influence of noise on the position of the maximum as a function of the cut-off frequency $u_{\mathrm{c}}$, and for different values of the signal-to-noise ratio $(S / N=$ $50,100,200)$. The results, for a line profile with $\delta \lambda=0.1 b$, $W=\left[\lambda_{0}-2 b, \lambda_{0}+2 b\right]$ and $r_{\mathrm{c}}=0.8$ (representative of our stars in Paper II), are shown in Fig. 9. The root mean square value of the noise on the $s_{\max }\left(u_{\mathrm{c}}\right)$ curve increases significantly if the signal-to-noise ratio decreases, and it slightly increases with the cut-off frequency. The modest increase of the solid line (without noise) is due to the wavelength binning, as follows from Fig. 7: for one particular value of the wavelength bin width, the error in the rotational velocity increases as a function of the cut-off frequency.
The average offset from the $s_{\max }\left(u_{\mathrm{c}}\right)$ curve due to noise is zero, but the root mean square value of the noise is significant, which implies that the uncertainty on the rotational velocity will be significant. To minimize this uncertainty, one may use the average of the values in the local maxima of the $s_{\max }\left(u_{\mathrm{c}}\right)$ curve instead of the value for just one cut-off frequency $u_{\mathrm{c}}$. In Fig. 10 we show that the uncertainty on this average is smaller than the uncertainty on the value for a single cut-off frequency. The solid line is the root mean square of the offset, due to noise, of the derived rotational velocity from the expected value, as a function of the signal-to-noise ratio, when using the average of the first four local maxima of the $s_{\max }\left(u_{\mathrm{c}}\right)$ curve. The dashed line is for the case where we only use the value of the first local maximum. In both cases the input parameters are the same as in Fig. 9.

If the relative central line intensity $r_{\mathrm{c}}$ is close to 1 (i.e., the line is very weak, or the rotational velocity is large), it will be difficult to distinguish the line from the continuum, in the presence of noise. Thus, it will be difficult to derive an accurate rotational velocity for such a line, also because the noise level in the Fourier transform is higher, because more wavelength points (with their noise) have been included. It is also difficult to derive an accurate rotational velocity for a noisy line profile with only a small number of wavelength bins. Figure 11 shows the minimum signal-to-noise ratio that is needed to find a rotational 


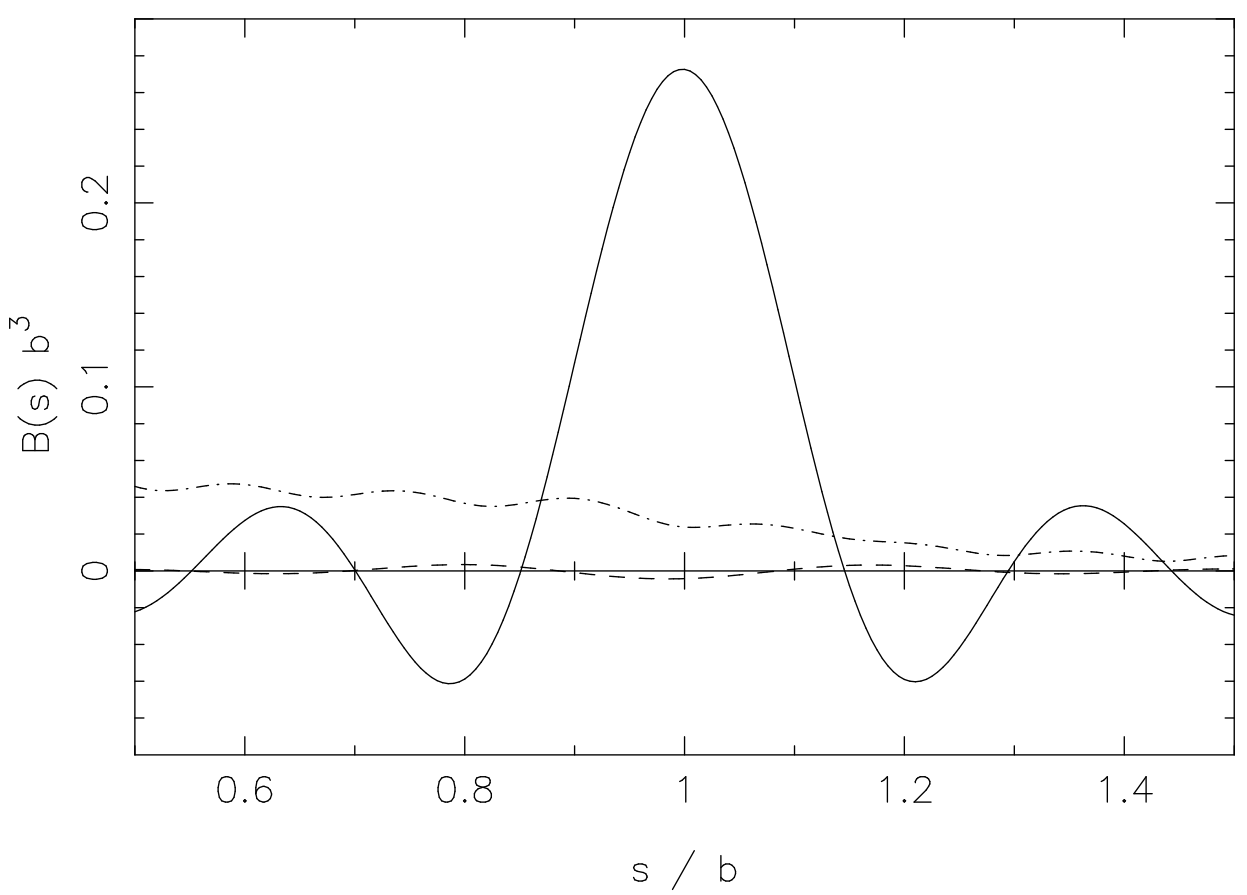

Fig. 8. The Fourier-Bessel transform $B(s)$ of a purely rotationally broadened profile without noise (solid line). The dashed line (approximately zero) denotes the average offset due to noise on the Fourier-Bessel transform, and the dot-dashed line denotes the root mean square of the noise, following from a Monte Carlo simulation on a line profile with Poisson noise. The parameters used for this simulation are: number of counts in the continuum $N=2500$ per wavelength bin, a relative central line intensity $r_{\mathrm{c}}=0.8$, a wavelength range $W=\left[\lambda_{0}-b, \lambda_{0}+b\right]$, a wavelength bin width $\delta \lambda=0.02 b$ and a cut-off frequency $u_{\mathrm{c}}=3.37 / b$

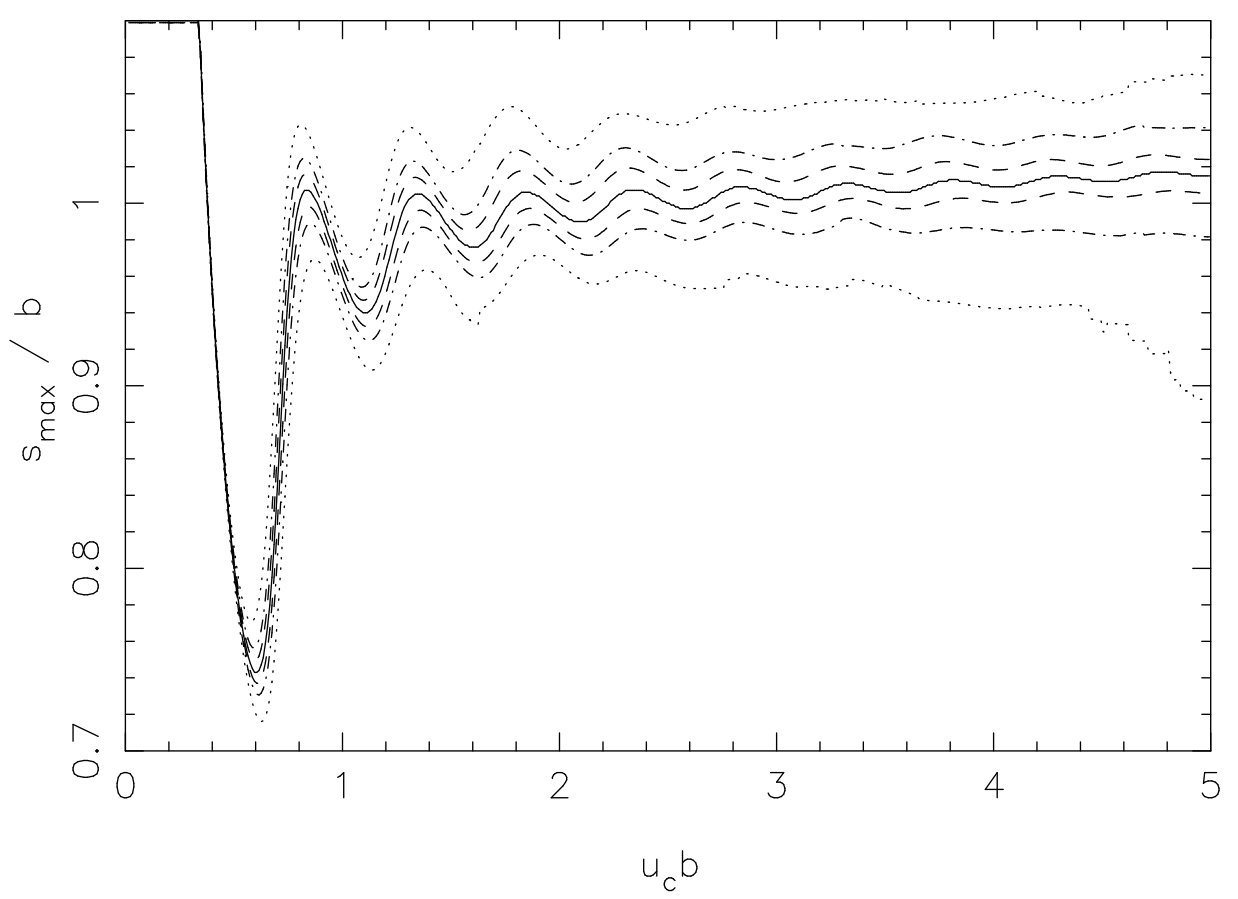

Fig. 9. The location of the maximum $s_{\max }$ of the Fourier-Bessel transform of a purely rotationally broadened profile without noise (solid line; identical to the curve in Fig. 4), in units of the rotational velocity parameter $b$, as a function of the cut-off frequency $u_{\mathrm{c}}$, in units of $b^{-1}$. The dotted line indicates the (root mean square) spread, due to noise, for a signal-to-noise ratio of $S / N=50$, the dot-dashed line for $S / N=100$ and the dashed line for $S / N=200$. We used a line profile with a wavelength bin width $\delta \lambda=0.1 b$, a wavelength range $W=\left[\lambda_{0}-2 b, \lambda_{0}+2 b\right]$ and a relative central line intensity $r_{\mathrm{c}}=0.8$ 


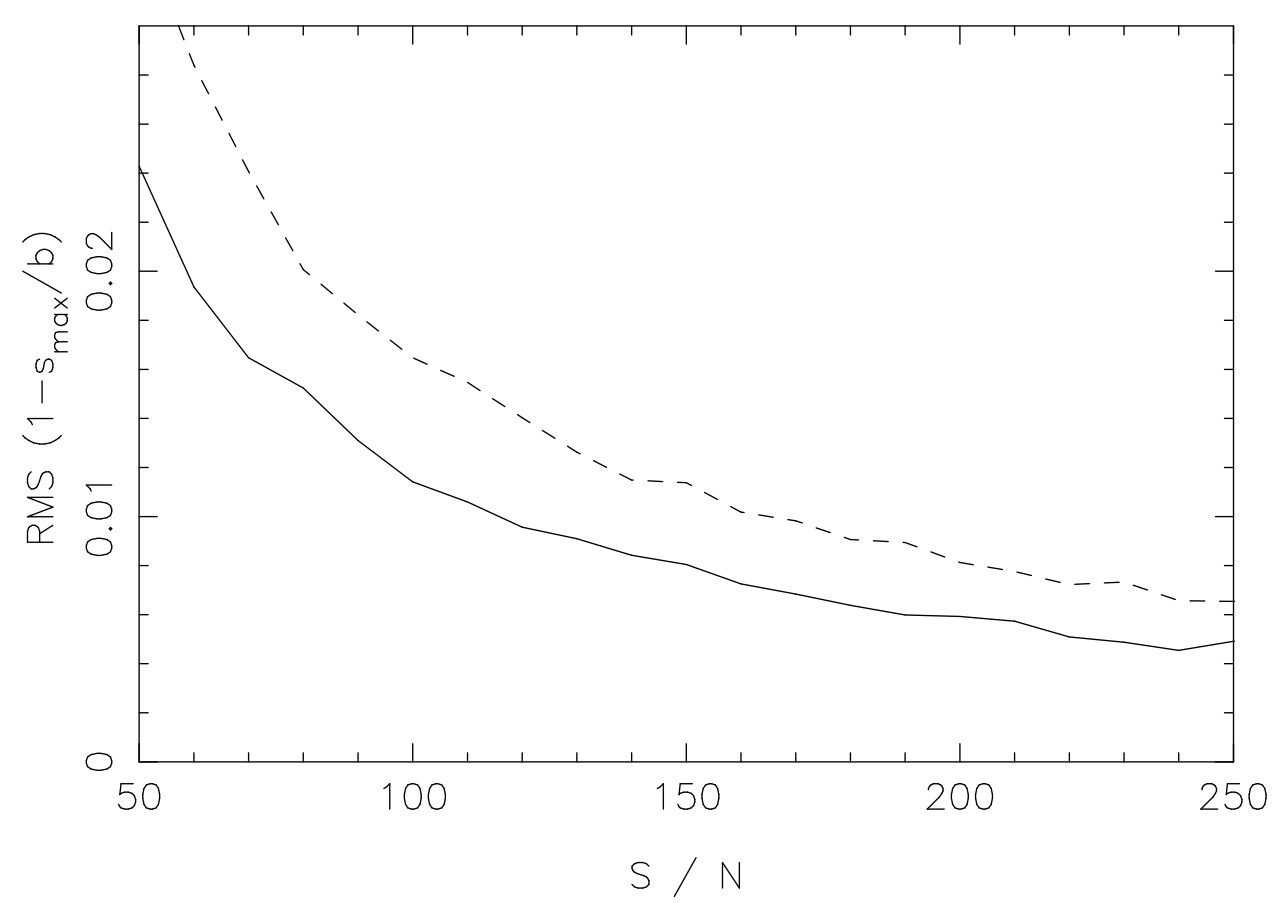

Fig. 10. The root mean square value of the offset, due to noise, from the rotational velocity parameter $b$, as a function of the signal-to-noise ratio. The solid line is for the case where we use the average of the first four local maxima of the $s_{\max }\left(u_{\mathrm{c}}\right)$ curve. The dashed line is for the case where we only use the value of the first local maximum. The line parameters used are the same as in Fig. 9

velocity with a maximum uncertainty due to noise of $1 \%$, for a specific wavelength bin width $\delta \lambda$ and relative central line intensity $r_{\mathrm{c}}$. For example: suppose we are interested in deriving rotational velocities up to $v \sin i=50 \mathrm{~km} / \mathrm{s}$ with a maximum uncertainty due to noise of $1 \%$. The absorption lines we have selected lie around $6000 \AA$, and have a relative central line intensity of about 0.8 (after convolution with the instrumental profile and with the rotational velocity profile of $50 \mathrm{~km} / \mathrm{s}$ ). In this case, the rotational velocity parameter $b$ is approximately 1 , for a velocity of $50 \mathrm{~km} / \mathrm{s}$. If we obtain spectra with a resolution of 60000 , i.e., $\delta \lambda=0.1 b$ for $v \sin i=50 \mathrm{~km} / \mathrm{s}$, we need a signal-tonoise ratio of at least 100 to find rotational velocities with a maximum uncertainty due to noise of $1 \%$.

The uncertainty, due to noise, on the rotational velocity is not significantly influenced by the wavelength range over which the Fourier transform is performed, as shown in Fig. 12.

\subsection{Intrinsic broadening and limb-darkening}

The intrinsic broadening of the spectral lines results in a systematic (mostly negative, Figs. 2 and 3 ) offset of the position of the maximum of the Fourier-Bessel transform from the rotational velocity parameter $b$ (Sect. 2.2). Hence, the Fourier-Bessel transformation method gives an equatorial rotational velocity with a systematic error caused by intrinsic broadening. The magnitude of this systematic error can be estimated from photospheric and spectral line parameters.

The influence of the limb-darkening coefficient on the position of the maximum is not clear from an analytical point of view (Sect. 2.4). The Fourier-Bessel transform of a spectral line influenced by limb-darkening has an additional contribution for values of $s$ less than the parameter $b$, as follows from Eq. (25) and can be seen in Fig. 6. This contribution shifts the maximum of the Fourier-Bessel transform to lower values. We have investigated this effect by taking the Fourier-Bessel transform of synthetic spectral lines with a constant limb-darkening coefficient. The simulations have been performed using a wavelength bin width $\delta \lambda / b$ of 0.07 and a frequency bin width $\delta u$ of 0.05 . Figure 13 shows the position of the maximum of the Bessel transform relative to the rotational velocity parameter $b$, as a function of the limb-darkening coefficient $\beta$ for four different values of the cut-off frequency. The cut-off frequencies $u_{\mathrm{c}}$ have been chosen to lie at local maxima of the function $s_{\max }\left(u_{\mathrm{c}}\right)$ in Fig. 4, where the position of the maximum of the Bessel transform is close to the rotational velocity parameter $b$, in the case of negligible limb-darkening (Sect. 2.3).

Hence, for one particular value of the limb-darkening coefficient, the location of the maximum of the FourierBessel transform approaches the rotational velocity parameter $b$, for large values of the cut-off frequency, in 


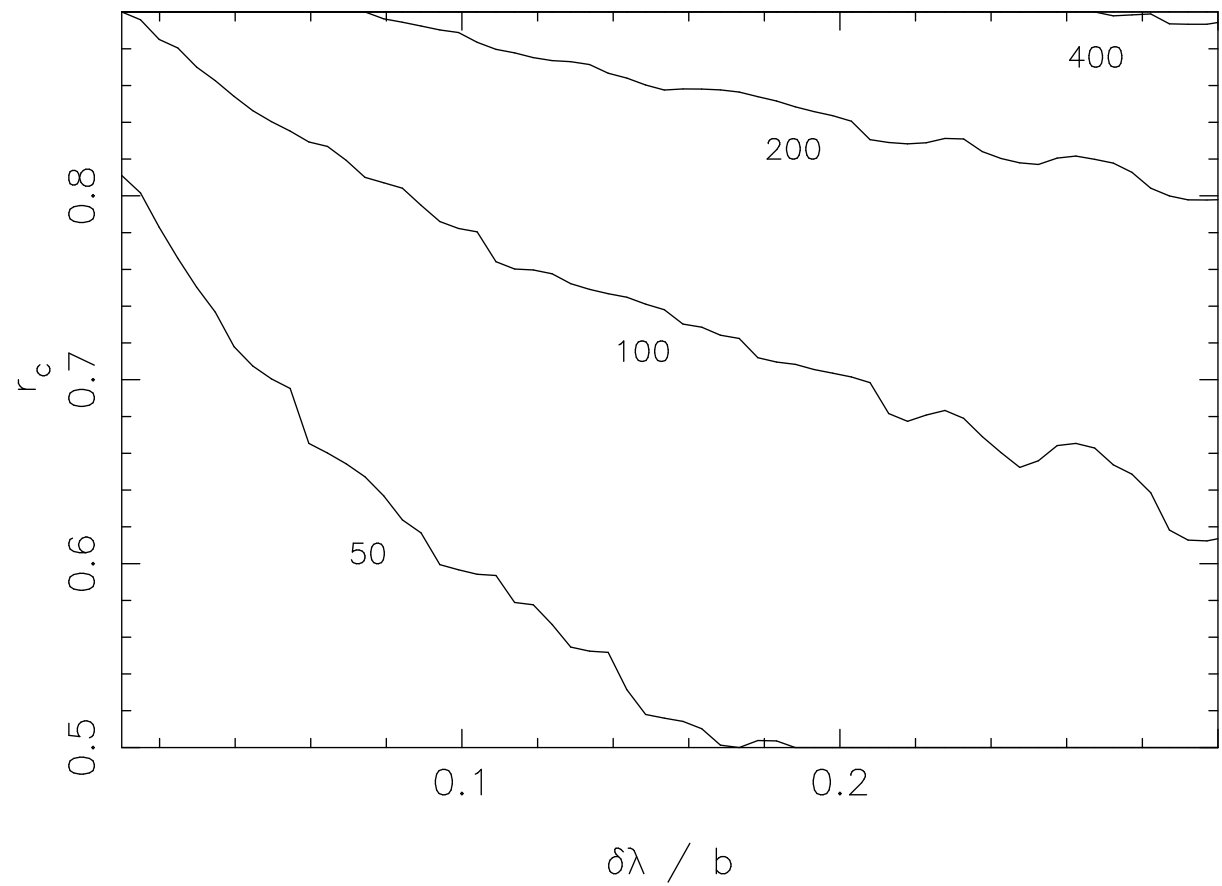

Fig. 11. Contour plot of the minimum signal-to-noise ratio needed to find a rotational velocity with a maximum uncertainty due to noise of $1 \%$, for a specific wavelength bin width $\delta \lambda$ and relative central line intensity $r_{\mathrm{c}}$. Lines are drawn for signal-to-noise ratios $S / N=50,100,200$, and 400

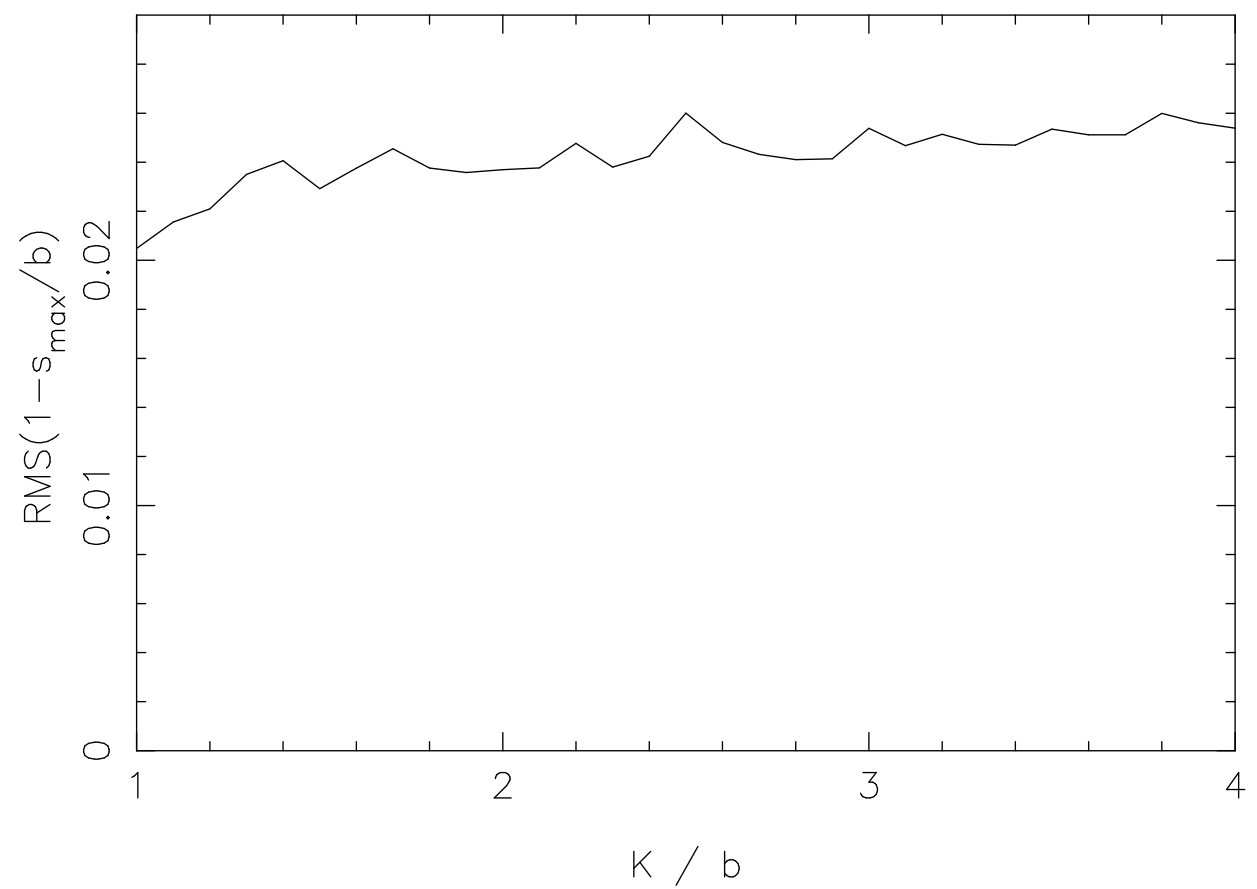

Fig. 12. The root mean square value of the offset, due to noise, from the rotational velocity parameter $b$, as a function of the wavelength range $W=\left[\lambda_{0}-K, \lambda_{0}+K\right]$. The signal-to-noise ratio is 50 , the wavelength bin width $\delta \lambda=0.1 b$, the relative central line intensity $r_{\mathrm{c}}=0.8$

agreement with the analytical expression for the FourierBessel transform of a rotationally broadened profile de- formed by limb-darkening (Eq. 25 and Fig. 6). The effect of limb-darkening on the location of the maximum of the 
Fig. 13. The location of the maximum of the Fourier-Bessel transform $s_{\max }$ as a function of the limb-darkening coefficient $\beta$. Different lines denote different cut-off frequencies $u_{\mathrm{c}}$ : the solid line is for $u_{\mathrm{c}} b=0.85$, the dashed line for $u_{\mathrm{c}} b=1.36$, the dot-dashed line for $u_{\mathrm{c}} b=1.68$, the dotted line for $u_{\mathrm{c}} b=2.37$

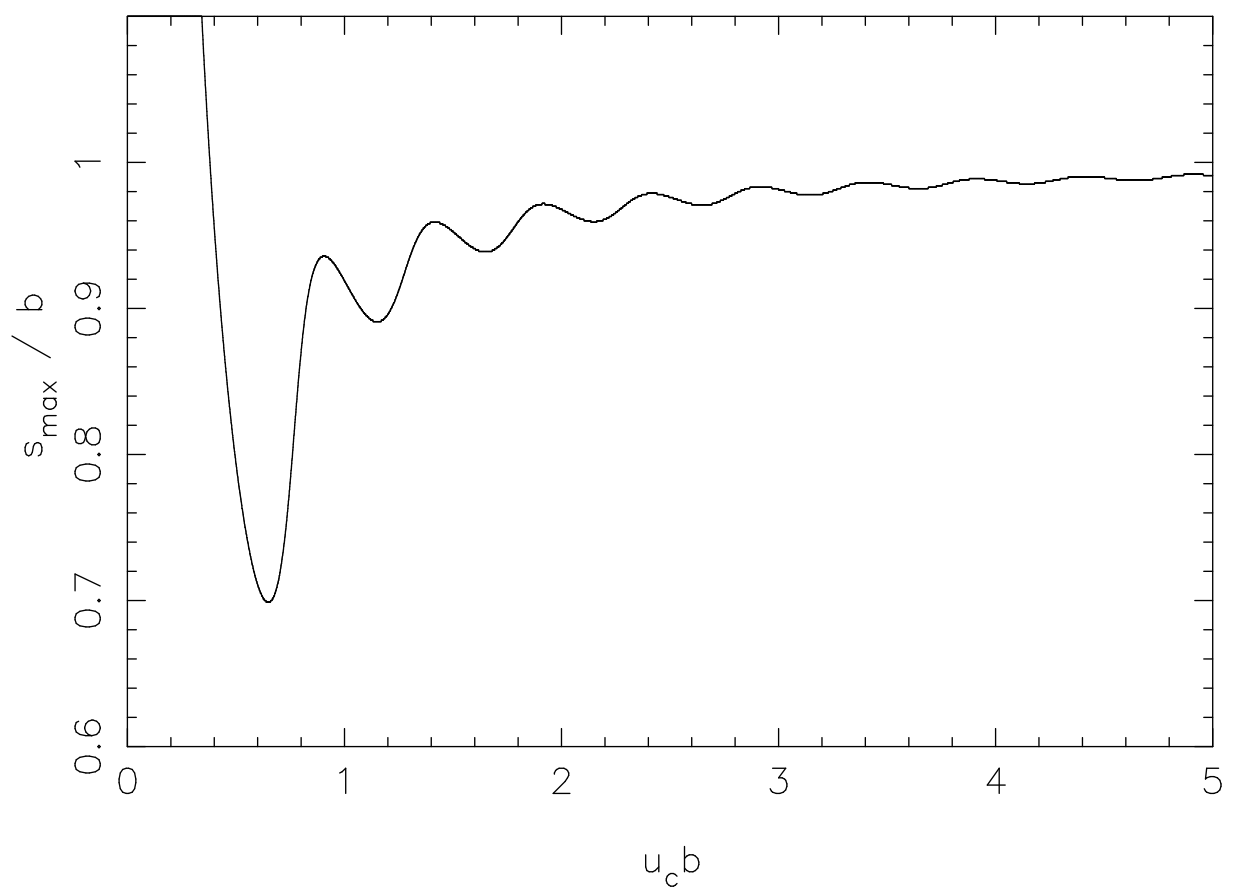

Fig. 14. The location of the maximum of the Fourier-Bessel transform $s_{\max }$ as a function of the cut-off frequency $u_{\mathrm{c}}$, for a rotationally broadened profile, deformed by limb-darkening. The limb-darkening coefficient is $\beta=1.0$

Bessel transform is considerable, but it can easily be recognized in the $s_{\max }\left(u_{\mathrm{c}}\right)$ curve by the increasing values of the local maxima, as shown in Fig. 14. So it is, in principle, possible to estimate the systematic error on the rotational velocity introduced by limb-darkening by comparing the values of the local maxima in the $s_{\max }\left(u_{\mathrm{c}}\right)$ curve with 


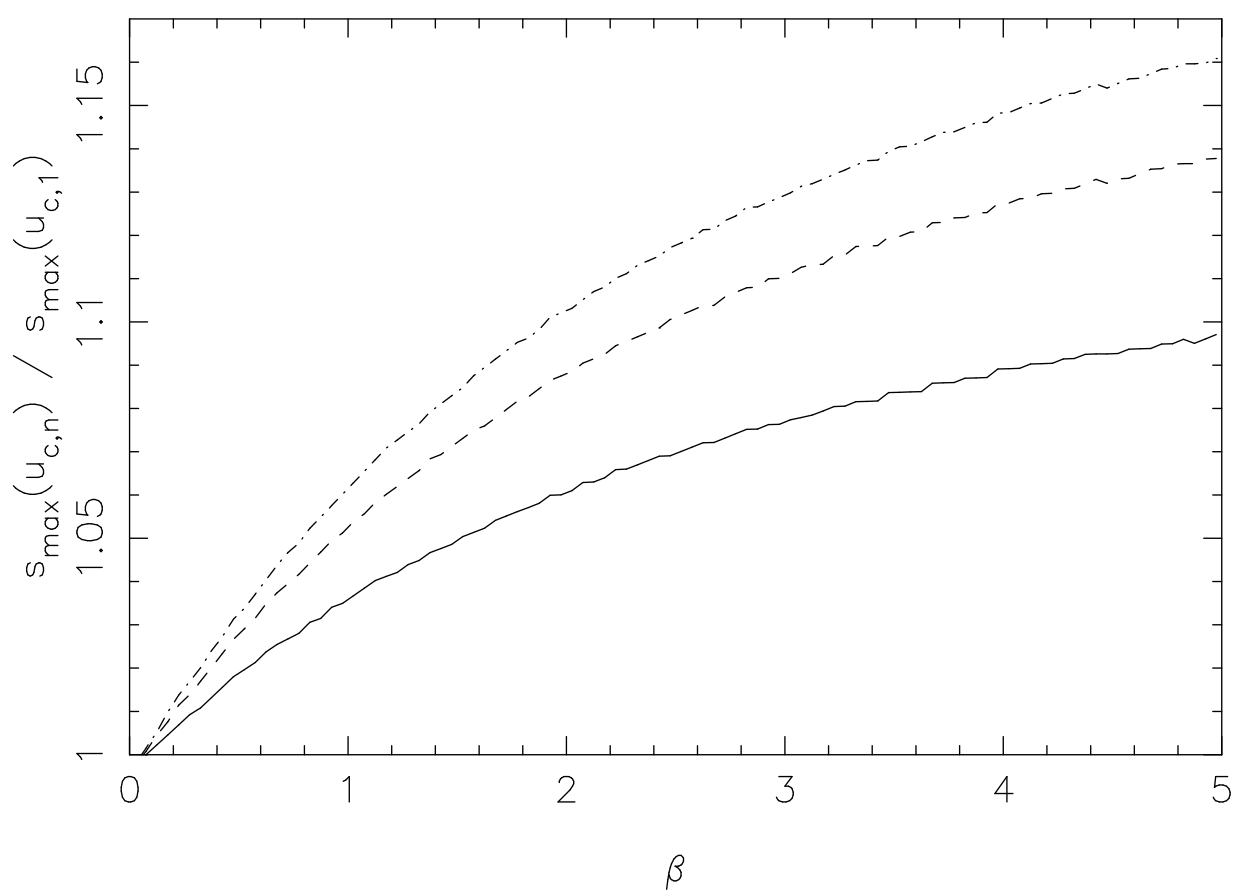

Fig. 15. The ratio $s_{\max }\left(u_{\mathrm{c}, n}\right) / s_{\max }\left(u_{\mathrm{c}, 1}\right)$ between the values at the $n^{\text {th }}$ and the first local maximum of the $s_{\max }\left(u_{\mathrm{c}}\right)$ curve, as a function of the limb-darkening parameter $\beta$

the values in Fig. 13. This is illustrated in Fig. 15, which shows the ratio between the values at the $n^{\text {th }}$ and the first local maximum of the $s_{\max }\left(u_{\mathrm{c}}\right)$ curve, as a function of the limb-darkening parameter $\beta$.

\subsection{Preparing the data}

The Fourier-Bessel transformation method is based on isolated symmetrical line profiles, with a well defined central wavelength, and a well defined continuum. In the process of preparing a spectral line $f(\lambda)$ for the Fourier-Bessel transformation method the following parameters have to be chosen: a central wavelength $\lambda_{0}$, a continuum level $f_{\mathrm{c}}(\lambda)$, and a wavelength range $\left[\lambda_{0}-K, \lambda_{0}+K\right]$ outside which $f(\lambda)$ is assumed to be equal to the continuum level $f_{\mathrm{c}}(\lambda)$. The transformation is performed on the line profile $I(\Delta \lambda)$, defined by

$$
I(\Delta \lambda)=1-\frac{f\left(\lambda_{0}+\Delta \lambda\right)}{f_{c}\left(\lambda_{0}+\Delta \lambda\right)}
$$

A wrong choice of the central wavelength, $\tilde{\lambda}_{0}$ instead of $\lambda_{0}$, results in a multiplication of the Fourier transform with a factor $\cos \left(2 \pi u\left(\tilde{\lambda}_{0}-\lambda_{0}\right)\right.$ ) (when only the real part of the transform is considered). In Figure 16 we show the location of the maximum $s_{\max }$ of the Bessel transform as a function of the difference $\Delta \lambda_{0}$ between the adopted and the real central wavelength $\tilde{\lambda}_{0}-\lambda_{0}$, in the case of a purely rotationally broadened profile. For comparison we show in the same figure the location of the maximum as a function of the wavelength bin width (Fig. 7), with the $x$-coordinate rescaled so that $\Delta \lambda_{0}$ is equivalent to $0.5 \delta \lambda$. Both curves are for a cut-off frequency $u_{\mathrm{c}}=1.36 / \mathrm{b}$. We conclude that as long as the central wavelength is not more off than half the bin width and not more off than $0.2 b$, the effect of making a wrong choice for the central wavelength is smaller than the effect of the binning itself.

If the continuum $f_{\mathrm{c}}(\lambda)$ is chosen too high or too low by a constant factor $1+\alpha$ (i.e., independent of the wavelength), so that $\tilde{f}_{\mathrm{c}}(\lambda)=(1+\alpha) f_{\mathrm{c}}(\lambda)$, the Fourier transform $\tilde{F}(u)$ becomes:

$$
\tilde{F}(u)=\frac{1}{1+\alpha}\left(F(u)+2 \alpha \frac{\sin (2 \pi u K)}{2 \pi u}\right)
$$

with $2 K$ being the wavelength range, over which the transform is performed. The additional sinc-function in the Fourier transform around frequency zero, gives an extra contribution to the Bessel transform. The influence of this contribution on the location of the maximum of the Bessel transform is shown in Fig. 17 for three different values of the continuum offset, for two different values of the cutoff frequency, and as a function of the wavelength range $W=\left[\lambda_{0}-K, \lambda_{0}+K\right]$ over which the Fourier transform is performed. This figure shows that the wavelength range should be chosen as large as possible, in order to minimize the uncertainty due to a misplaced continuum. However, the wavelength range should not be chosen so large that it is contaminated by the contribution from neighbouring lines. The cut-off frequency should also be chosen as large 


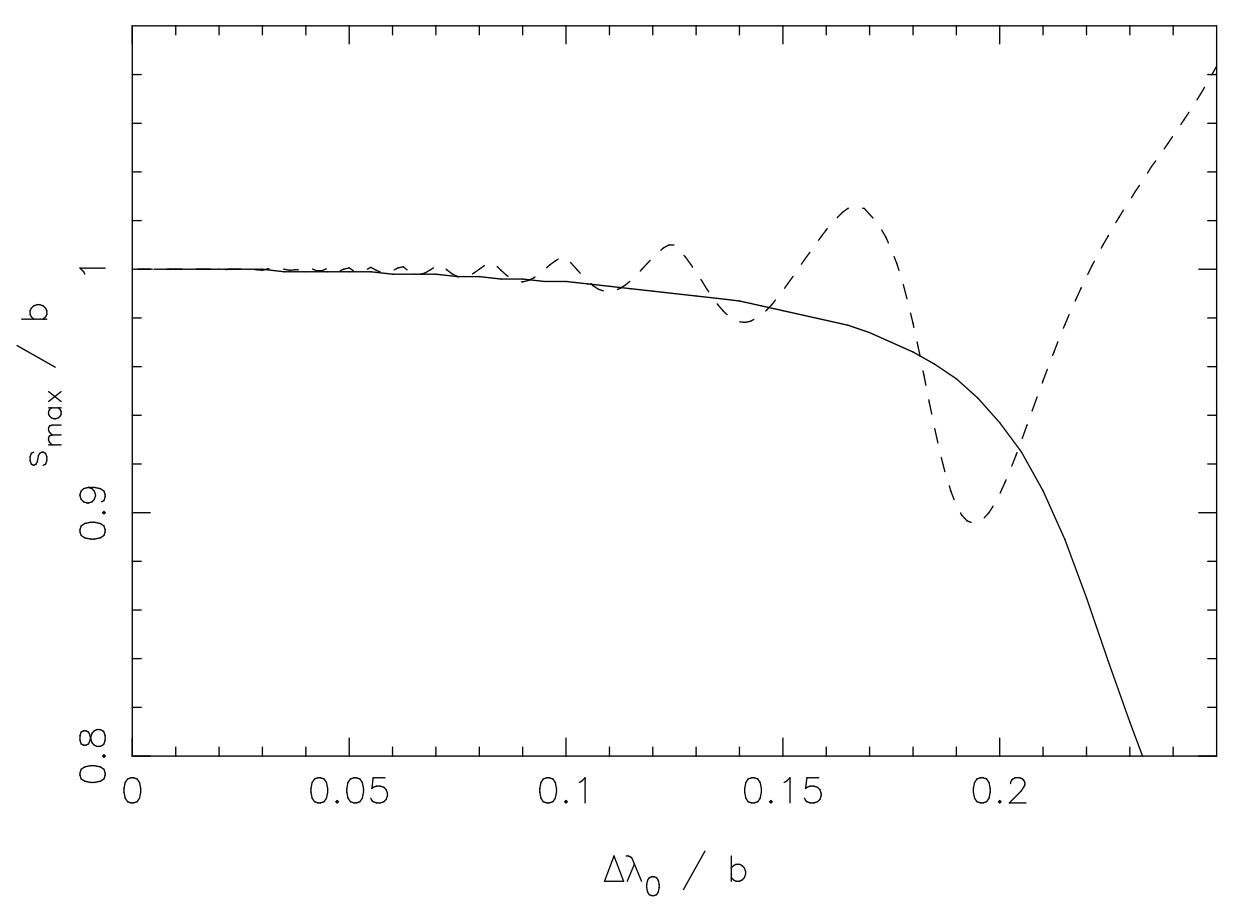

Fig. 16. The location of the maximum $s_{\max }$ of the Fourier-Bessel transform as a function of the difference $\Delta \lambda_{0}$ between the chosen central wavelength $\tilde{\lambda}_{0}$ and the real central wavelength $\lambda_{0}$ (solid curve). The dashed line shows $s_{\max }$ as a function of the wavelength bin width $\delta \lambda$ from Fig. 7

as possible, because the uncertainty on the rotational velocity, due to a misplacement of the continuum, decreases for increasing cut-off frequencies.

\section{Discussion}

Gray (1988) has extensively used the Fourier transforms of line profiles to study line broadening. His approach consists in making fits of the Fourier transforms to functions derived for detailed model line profiles broadened by rotation. The relative advantage of this approach and the FBT method depends on the application one has in mind. Gray's method shows with the Fourier-Bessel transformation method the advantage that it can distinguish rotational broadening from other broadening mechanisms; in addition, it allows one to infer detailed information on other broadening mechanisms at will (something that the Fourier-Bessel transformation method does not). If one is only interested in rotation, the Fourier-Bessel transformation method has the advantage that no model parameters are needed and allows an easy way to measure rotational velocities for large samples of stars.

The Fourier-Bessel transformation method can give projected equatorial rotational velocities which, for medium strong lines observed with high signal-to-noise and high spectral resolution, can reach an (internal) accuracy of several percent. The method allows a seperation of rotational broadening and other broadening mechanisms, such as Gaussian and Lorentzian broadening. The spectral resolution limits the accessible velocity range on the low side: we can find velocities as small as $2 c \Delta \lambda / \lambda_{0}$ with an uncertainty of $10 \%$ (Sect. 2.5). For sufficiently high spectral resolution the method allows the measurement of rotational velocities as small as $0.8 c \Delta \lambda_{\mathrm{D}} / \lambda_{0}$, with a similar uncertainty, in case Doppler broadening (other than rotation) is the dominant broadening mechanism. (Sect. 2.2.1). If damping is the dominant broadening mechanism (but this is never the case for medium strong lines encountered in normal stellar atmospheres) rotational velocities can be measured with $10 \%$ accuracy down to $0.3 c \Delta \lambda_{\mathrm{H}} / \lambda_{0}$ (see Sect. 2.2.2). The maximum of the rotational velocity range that can be studied with this method depends on the signal-to-noise ratio of the spectrum, the intrinsic depth of the line, the line density (line blending) and the spectral resolution of the line profile (Sect. 3.1).

The velocities derived with the FBT method are likely affected by systematic effects; an important effect is limb darkening. For a standard limb darkening parameter of $\beta$ $=0.6$ (the value for a grey atmosphere in radiative equilibrium), the FBT method, as presented by us, (i.e. not including limb darkening), underestimates the rotational velocity systematically by $\leq 5 \%$ (see Sect. 3.2 )

The FBT method does not provide detailed modelling of complicated broadening mechanisms, such as anisotropic macroscopic velocity fields in the stellar atmosphere. Such detailed study, in which the largest 

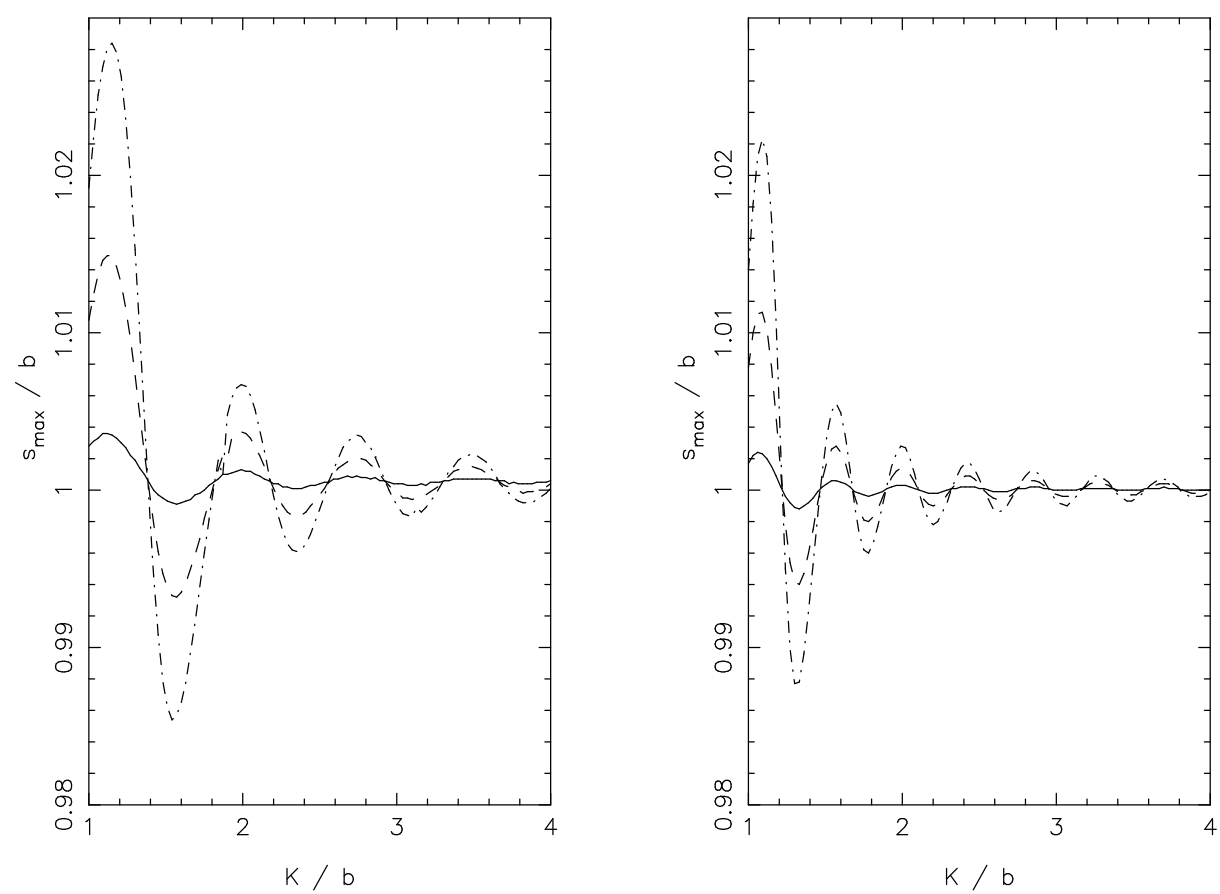

Fig. 17. The location of the maximum $s_{\max }$ of the Fourier-Bessel transform as a function of the wavelength range $W=\left[\lambda_{0}-K, \lambda_{0}+K\right]$, for three different values of the continuum offset: the solid line is for $\alpha /\left(1-r_{\mathrm{c}}\right)=0.01$, the dashed line for $\alpha /\left(1-r_{\mathrm{c}}\right)=0.05$, and the dot-dashed line for $\alpha /\left(1-r_{\mathrm{c}}\right)=0.1$. Figure a) is for a cut-off frequency $u_{\mathrm{c}}=1.36 / b$, Fig. $\left.\mathbf{b}\right)$ for $u_{\mathrm{c}}=4.38 / b$

accuracy is required can be performed by fitting of model parameters to the Fourier transform of the spectral line (see Gray 1988). The FBT method is therefore best suited for statistical investigations of large samples of stars, for which medium spectral resolution and signal-to-noise data are available, in which the detailed properties of individual stars are not the focus of attention, and the highest accuracy is not required. An example of such a study is presented in Paper II.

\section{References}

Böhm K., 1952, Zeit. f. Astrophysik 30, 117
Carrol J.A., 1933, MNRAS 93, 478

Deeming T.J., 1977, Ap\&SS 46, 13

Gray D.F., 1988, Lectures on Spectral-Line Analysis: F, G and K stars, The Publisher, Arva, Ontario, Canada

Groot, P.J., Piters, A.J.M., Van Paradijs, J., 1996, (Paper II), A\&A, (submitted)

Oberhettinger, F., 1970, Handbook of Mathematical Functions. In: Abramowitz M., Stegun I.A. (eds.). Dover Publ., NY, U.S.A., Chapter 15

Shajn G., Struve O., 1920, MNRAS 89, 221 